\title{
Nonlinear caging in all-bands-flat lattices
}

\author{
Carlo Danieli $\odot,{ }^{1,2}$ Alexei Andreanov $\odot,{ }^{2,3}$ Thudiyangal Mithun $\odot,{ }^{2,4}$ and Sergej Flach ${ }^{2,3}$ \\ ${ }^{1}$ Max Planck Institute for the Physics of Complex Systems, Dresden D-01187, Germany \\ ${ }^{2}$ Center for Theoretical Physics of Complex Systems, Institute for Basic Science (IBS), Daejeon 34126, Korea \\ ${ }^{3}$ Basic Science Program (IBS School), Korea University of Science and Technology (UST), Daejeon 34113, Korea \\ ${ }^{4}$ Department of Mathematics and Statistics, University of Massachusetts, Amherst, Massachusetts 01003-4515, USA
}

(Received 30 April 2020; accepted 20 July 2021; published 17 August 2021)

\begin{abstract}
We study the impact of classical short-range nonlinear interactions on transport in lattices with no dispersion. The single-particle band structure of these lattices contains flat bands only, and cages noninteracting particles into compact localized eigenstates. We demonstrate that there always exist local unitary transformations that detangle such lattices into decoupled sites in dimension 1. Starting from a detangled representation and inverting the unitary transformations, we arrive at the all-bands-flat generator for single-particle Hamiltonians in one dimension, which is also straightforwardly generalized to higher dimensions. The entangling unitary transformations are parametrized by sets of angles. For a given member of the set of all-bands-flat, additional short-range nonlinear interactions destroy caging in general, and induce transport. However, fine-tuned subsets of the unitary transformations allow caging to be completely restored. We derive the necessary and sufficient fine-tuning conditions for nonlinear caging, and we provide computational evidence of our conclusions for one-dimensional systems.
\end{abstract}

DOI: 10.1103/PhysRevB.104.085131

\section{INTRODUCTION}

Understanding the impact of interactions on single-particle localized states has been one of the most intriguing quests of the past decades in condensed-matter physics. Classical and quantum approaches may yield seemingly distinct outcomes while starting from the same single-particle localization. One notable example concerns the impact of interactions on Anderson localization-i.e., the exponential localization of all single-particle states due to uncorrelated disorder and the confinement of noninteracting particles over finite portions of the lattice $[1,2]$. Weakly interacting quantum particles show a finite-temperature transition from a thermalized to a manybody localized phase [3-5]. Classical interactions instead predict finite heat and particle conductivity at arbitrarily small temperatures, and related indefinite subdiffusive wave-packet spreading [6,7].

In translationally invariant networks, destructive interference can fully localize subfamilies of single-particle eigenstates within a finite portion of the lattice. These eigenstates-dubbed compact localized states (CLS) - have macroscopically degenerate eigenenergies and form dispersionless (or flat) Bloch bands in band structures containing otherwise dispersive bands. While they were originally used

Published by the American Physical Society under the terms of the Creative Commons Attribution 4.0 International license. Further distribution of this work must maintain attribution to the author(s) and the published article's title, journal citation, and DOI. Open access publication funded by the Max Planck Society. to study degenerate ferromagnetic ground states $[8,9]$, flatband lattices have drawn a lot of theoretical attention ever since, and compact localized states have been observed experimentally in several settings, from ultracold atomic gases to photonics; for an overview on recent advances, see Refs. [10-12]. Flatband networks can be viewed as fine-tuned submanifolds in a suitably defined space of Hamiltonian tightbinding networks.

Remarkably, flatband networks can be further fine-tuned in order to flatten remaining dispersive bands, all the way down to an important subclass of the family of flatband lattices which possess only perfectly flat Bloch bands. The absence of single-particle dispersion yields the strict confinement of noninteracting particles within the lattice. This was first shown in a two-dimensional lattice structure in the presence of a magnetic field which was fine-tuned to reach a time-reversal invariant model [13], and was dubbed Aharonov-Bohm caging $(\mathrm{AB})$, while the compact eigenstates were referred to as caged states. This path to reach single-particle caging has been extended further in the past decade [14-18] and it has been experimentally realized using photonic lattices $[19,20]$ and qubit nanocircuits [21], among others. Interestingly, the introduction of a magnetic field, which in general does break time reversal, is not of essence and not needed at all, as we will show below. The route to zero dispersion and caging via magnetic fields leads to one model realization among whole manifolds of systems that lack dispersion. We coin such systems all bands flat (ABF) lattices.

The impact of interactions on the ABF single-particle caging has been studied in a number of attempts in both classical and quantum regimes. A notable setup for these studies has been the one-dimensional (1D) diamond (rhombic) $\mathrm{ABF}$ 
chain. In this case, while local Kerr nonlinear interactions preserve caging [22,23], Hubbard interactions induce transporting bound states of two particles simultaneously [24]. The study of quantum interactions for caged noninteracting particles has been further developed in different $\mathrm{ABF}$ geometries, including the 1D Creutz lattice [25-30] and the 2D Dice lattice [31].

In this work, we study classical nonlinear interactions in ABF networks. We will obtain conditions under which an additional fine-tuning in the manifold of ABF networks leads to a complete caging in the presence of nonlinear interactions. We will show that in the absence of interactions, proper local unitary transformations lead to a complete detangling of the network into decoupled sites for 1D systems - a fact that holds for any number of bands and provides a systematic (in any lattice dimension) and exhaustive (at least in 1D) generator for ABF lattices. We employ these unitary transformations to show that nonlinear interactions in general break the singleparticle caging and result in transport and delocalization. We then obtain necessary and sufficient fine-tuning conditions for the nonlinear interaction to preserve caging. The condition is then tested for $1 \mathrm{D}$ networks with $v=2,3$, 4 flatbands, which will include previously studied diamond chain $(v=3)$ examples, for which the nonlinear caging was previously found $[22,23]$. We further present extensions to $2 \mathrm{D}$ nonlinear caging models. The intricate related caging features arising from quantum interactions will be unfolded in a subsequent work [32].

\section{SINGLE-PARTICLE CAGING}

Let us consider the unitary evolution of a one-dimensional tight-binding problem with nearest neighbor unit-cell coupling:

$$
i \dot{\psi}_{n}=-H_{0} \psi_{n}-H_{1} \psi_{n+1}-H_{1}^{\dagger} \psi_{n-1} .
$$

For any $n \in \mathbb{Z}$, each component of the complex vector $\psi_{n}=$ $\left(\psi_{n, 1}, \ldots, \psi_{n, v}\right)^{T}$ represents a site of the periodic lattice, and therefore $\psi_{n}$ represents its unit cell. The profile of the network is defined by the square matrices $H_{0}, H_{1}$. The transformation $\psi_{n}=x_{n} e^{-i E t}$ yields the eigenvalue problem associated with Eq. (1), and then the Bloch solution $x_{n}=e^{i \mathbf{k} n} y_{k}$ defined for the wave-vector $\mathbf{k}$ gives rise to the band structure $\left\{E_{j}(\mathbf{k})\right\}_{j=1}^{v}$ of Eq. (1).

In this work, we focus on $\mathrm{ABF}$ networks where all bands $E_{j}$ are independent of $\mathbf{k}$-hence all bands are flat. The collapse of the single-particle spectrum into several flatbands and the absence of dispersive states is coined "caging." Any compact initial condition remains confined within a finite (compact) subvolume of the network $\psi_{n}(t) \neq 0$ for $1 \leqslant n \leqslant M$ and $\psi_{n}(t)=0$ otherwise, for all $t \in \mathbb{R}$.

In short-range flatband networks, the eigenstates associated with the flatband can always be recast as spatially compact [33]. Using this as a starting point, we prove the following:

Theorem: Any one-dimensional $v \geqslant 2$ all bands flat network (1) with short-range hopping can be recast into a fully decoupled lattice,

$$
i \dot{\phi}_{n}=H_{R} \phi_{n}, \quad H_{R}=\operatorname{diag}\left(E_{1}, E_{2}, \ldots, E_{\nu}\right)
$$

by a translationally invariant unitary transformation $\mathcal{U}$,

$$
\begin{aligned}
\mathcal{U} \Psi=\Phi, \quad \Psi & =\left(\psi_{1}, \ldots, \psi_{N}\right), \Phi=\left(\phi_{1}, \ldots, \phi_{N}\right), \\
\mathcal{U} & =\prod_{l=1}^{L} \mathcal{U}_{l}, \quad \mathcal{U}_{l}=\sum_{n=1}^{N} u_{n},
\end{aligned}
$$

where $u_{n}$ are local commuting unitary transformations: unit cell basis changes and unit cell redefinitions; and $N$ is the total number of unit cells.

The main idea behind this result is that in one dimension there always exist a sequence of local unitary transformations each redefining the unit cells which recast all the flatband compact eigenstates within a single unit cell-revealing, therefore, the orthogonality of the eigenstates. Therefore an equivalent statement of the theorem is that the $d=1 \mathrm{ABF}$ lattices always have orthonormal compact localized eigenstates. This statement is far from trivial: compact localized states of a flatband that coexists with other dispersive bands are not necessarily orthogonal, e.g., stub lattice [34-36] or the notable Lieb lattice [11] provide counterexamples. Furthermore in the latter case compact states are not even complete (similarly to kagome and pyrochlore lattices [37]). The detailed proof can be found in Appendix A 2. For the specific ABF case of nearest-neighbor unit-cell coupling in Eq. (1) it follows that compact localized states occupy two unit cells, and the detangling procedure involves one local unitary transformation which decouples the lattice into noninteracting $v$-mers. One subsequent unit cell redefinition and one more unitary transformation which diagonalizes the $v$-mer results in the detangled form (2). Increasing the hopping range results in the corresponding increase of the number of nested unit cell redefinitions and local unitary transformations. In what follows, we refer to the basis where the Hamiltonian is diagonal as detangled basis.

We conjecture that this result holds in higher dimensions, and any short-range $\mathrm{ABF}$ lattice in any dimensions is equivalent to decoupled sites up to a local unitary transformation. Note that the inverse is always true. Starting from a detangled set of sites, reversing the detangling procedure $\phi_{n} \longmapsto \psi_{n}$ yields a generator of any one dimensional $\mathrm{ABF}$ lattice, and of a plethora (if not any) of higher-dimensional ABF lattices, for any finite number of bands $v$. In the simplest case of nearest-neighbor unit-cell coupling and a fixed unit cell redefinition one ABF manifold has dimension 2( $\left.v^{2}-1\right)$ since it is controlled by two unitary transformations (we removed the trivial global phases as irrelevant parameters). The manifold contains the detangled model. Since there are $2^{v}-2$ possible unit cell redefinitions, there are as many different ABF manifolds, all originating from one and the same detangled model. Extending to a nested sequence of $\mu$ unit cell redefinitions increases the hopping range to distance $\mu$ and dramatically increases the number of ABF manifolds as well as their dimensions. This scheme completes a list of several other generator schemes introduced in recent years [38-43] which instead were focused on flatband networks supporting both flat and dispersive bands.

The manifold of ABF lattices generated with this method include the known examples of Aharonov-Bohm caging, e.g., the Creutz ladder (as discussed below) and diamond chain in 

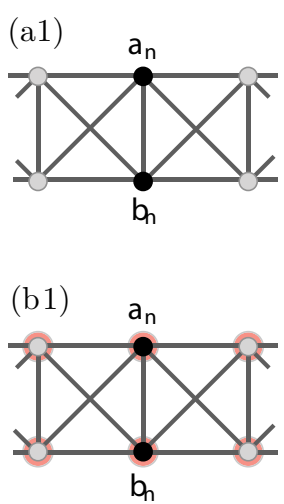

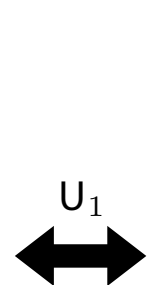

(a2)

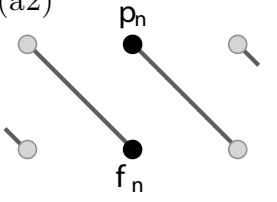

(202)

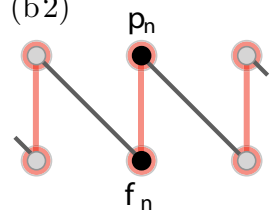

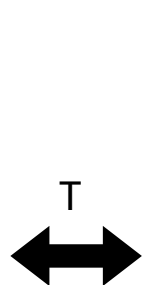

(a3)
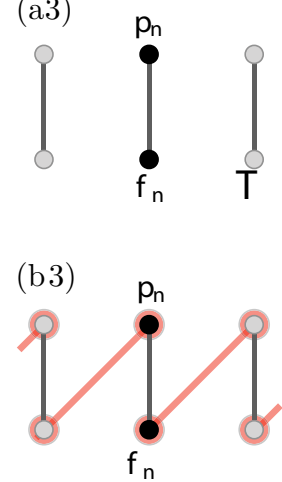

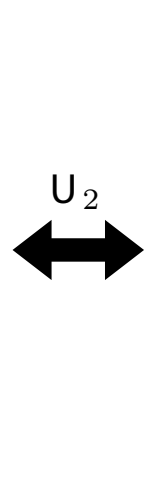

$(\mathrm{a} 4)$

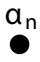

$\beta_{n}$

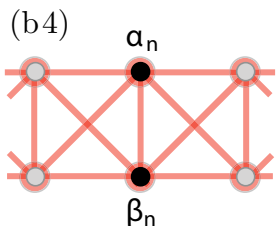

FIG. 1. Schematic representation of the unit-cell redefinition for a $v=2$ ABF lattice. In each panel, the black dots label the chosen unit cell. The solid gray lines represent the linear hopping terms; the red shaded lines represent the interaction terms. (a1)-(a4) Noninteracting regime. (b1)-(b4) Interacting regime.

a fine-tuned magnetic field $[13,14,25]$. On the other hand it is not at all obvious, and likely also not very relevant, whether any such generated $\mathrm{ABF}$ lattice is equivalent to some tightbinding lattice in a properly fine-tuned magnetic field, even if one allows for artificial fluxes in the spirit of the Haldane model [44].

Let us visualize this procedure for the simplest case of $v=2$ networks in Figs. 1(a1)-1(a4), with the canonical coordinates $\psi_{n}=\left(a_{n}, b_{n}\right)$ and the detangled coordinates $\phi_{n}=$ $\left(\alpha_{n}, \beta_{n}\right)$. The detangling procedure $\mathcal{U}: \mathcal{U} \psi_{n}=\phi_{n}$ unfolds in three steps

$$
\left(\begin{array}{c}
a_{n} \\
b_{n}
\end{array}\right) \stackrel{U_{1}}{\longmapsto}\left(\begin{array}{c}
p_{n} \\
f_{n}
\end{array}\right) \stackrel{T}{\longmapsto}\left(\begin{array}{c}
p_{n} \\
f_{n}
\end{array}\right) \stackrel{U_{2}}{\longmapsto}\left(\begin{array}{c}
\alpha_{n} \\
\beta_{n}
\end{array}\right)
$$

with the alternation of two unitary transformations $U_{1}$ and $U_{2}$ and one relabeling of the lattice sites $T$ :

$$
U_{i}=e^{i \theta_{i}}\left(\begin{array}{cc}
z_{i} & w_{i} \\
-w_{i}^{*} & z_{i}^{*}
\end{array}\right), \quad T:\left\{\begin{array}{l}
p_{n} \longmapsto p_{n}, \\
f_{n} \longmapsto f_{n-1} .
\end{array}\right.
$$

The complex numbers $z_{i}, w_{i}$ are constrained with $\left|z_{i}\right|^{2}+$ $\left|w_{i}\right|^{2}=1$. Without loss of generality, the two flatband energies can be locked at $E= \pm 1$. We then parametrize the matrices $H_{0}, H_{1}$ for $v=2 \mathrm{ABF}$ networks in Eq. (1) as

$$
\begin{gathered}
H_{0}=\Gamma_{0}\left(\begin{array}{cc}
\left|z_{1}\right|^{2}-\left|w_{1}\right|^{2} & -2 z_{1} w_{1} \\
-2 z_{1}^{*} w_{1}^{*} & \left|w_{1}\right|^{2}-\left|z_{1}\right|^{2}
\end{array}\right), \\
H_{1}=\Gamma_{1}\left(\begin{array}{cc}
z_{1} w_{1}^{*} & z_{1}^{2} \\
-\left(w_{1}^{*}\right)^{2} & -z_{1} w_{1}^{*}
\end{array}\right),
\end{gathered}
$$

with $\Gamma_{0}=\left|w_{2}\right|^{2}-\left|z_{2}\right|^{2}$ and $\Gamma_{1}=2 z_{2} w_{2}$ (see Appendix A 3 for details). The resulting manifold of $v=2 \mathrm{ABF}$ lattices in Eqs. (5) and (6) includes the notable Creutz lattice [25], which is obtained for $z_{1}=z_{2}=w_{2}=1 / \sqrt{2}$ and $w_{1}=i / \sqrt{2}$. Our manifold also includes a lower-dimensional submanifold of lattices related to the Creutz lattice via a gauge transformation $[30,45]$.

The result that any $1 \mathrm{D}$ ABF lattice is unitarily equivalent to a set of decoupled sites provides a powerful framework for analysis of $\mathrm{ABF}$ networks, for example their transport properties, in presence of various perturbations, in particular interactions/nonlinearities.

\section{NONLINEAR INTERACTIONS: SUBDIFFUSION AND FINE-TUNED CAGING}

Any linear ABF network cages any spatially localized initial excitation: the excitation remains confined as time evolves; in particular, excitations separated by strictly zero unit cells are forbidden to communicate. An important question is the fate of this caging behavior in the presence of interactions. We will address this question in the following way: A given linear $\mathrm{ABF}$ Hamiltonian is a member of a manifold of ABF Hamiltonians linked together by (local) unitary transformations. We pick one of the members of that manifold, add local nonlinear interactions, and we need to determine whether caging is destroyed. For that, we transform the chosen member into the detangled basis, inspect the transformed nonlinear interactions, and arrive at necessary and sufficient conditions for nonlinear caging.

Let us add nonlinear terms to the Schrödinger equation (1) which result, e.g., from a mean-field approximation to a bosonic many-body interacting system. For convenience, we choose the local Kerr-like nonlinearity. This choice is not essential for the following arguments, and is made for convenience only. Equation (1) becomes

$$
i \dot{\psi}_{n}=-H_{0} \psi_{n}-H_{1} \psi_{n+1}-H_{1}^{\dagger} \psi_{n-1}+U F\left(\left|\psi_{n}\right|^{2}\right) \psi_{n} .
$$

Here $F\left(\left|\psi_{n}\right|^{2}\right)$ is a diagonal matrix with nonzero elements $F_{\mu, \mu} \equiv\left|\psi_{n, \mu}\right|^{2}$. The above Gross-Pitaevskii-type lattice equations are generated by the Hamiltonian

$$
\begin{gathered}
i \dot{\psi}_{n}=\nabla_{\psi_{n}^{*}} \mathcal{H}_{G}, \quad \hat{\mathcal{H}}_{G}=\hat{\mathcal{H}}_{0}^{G}+\hat{\mathcal{H}}_{1}^{G}, \\
\mathcal{H}_{0}^{G}=-\sum_{n \in \mathbb{Z}}\left[\frac{1}{2}\left(\psi_{n}^{* T} H_{0} \psi_{n}\right)+\left(\psi_{n}^{* T} H_{1} \psi_{n+1}\right)+\text { H.c. }\right], \\
\mathcal{H}_{1}^{G}=\frac{U}{2} \sum_{n \in \mathbb{Z}} F^{2}\left(\left|\psi_{n}\right|^{2}\right) .
\end{gathered}
$$

The coordinate redefinition $\psi_{n} \longmapsto \phi_{n}$ to Eq. (7) decouples the quadratic part. The local nonlinear terms in the original representation $\psi_{n}$ become nonlocal in the new coordinates $\phi_{n}$ of the detangled basis. The evolution equation (7) in the new representation reads

$$
i \dot{\phi}_{n}=H_{R} \phi_{n}+g \mathcal{P}\left(\left\{\phi_{n}\right\}\right) .
$$


$H_{R}$ is the diagonal matrix in Eq. (2) and $\mathcal{P}$ is a homogeneous polynomial of degree 3 in $\left\{\phi_{n}\right\}$ :

$$
\mathcal{P}\left(\left\{\phi_{n}\right\}\right)=\sum_{m b ; k c, l d} V_{n a, m b ; k c, l d} \phi_{m, b}^{*} \phi_{k, c} \phi_{l, d} .
$$

Here $n$ labels unit cells and $a$ labels sites in the unit cell $n$. The matrix elements $V_{n a, m b ; k c, l d}$ define a nonlinear interaction network in the detangled basis with the corresponding Hamiltonian,

$$
\mathcal{H}_{1}^{G}=\frac{U}{2} \sum_{n a, m b ; k c, l d} V_{n a, m b ; k c, l d} \phi_{n, a}^{*} \phi_{m, b}^{*} \phi_{k, c} \phi_{l, d} .
$$

This nonlinearity has several properties that we use later: the range of this interaction is strictly finite, spanning only a finite number of unit cells, since the Kerr-like nonlinearity is local and the unitary transformation defining the detangled basis is a composition of a finite number of local unitary transformations. The nonlinearity (10) is translationally invariant and therefore $\mathcal{H}_{1}^{G}$ contains the term $\phi_{n, a}^{*} \phi_{m, b}^{*} \phi_{k, c} \phi_{l, d}$ along with its translated copies $\phi_{n+p, a}^{*} \phi_{m+p, b}^{*} \phi_{k+p, c} \phi_{l+p, d}$ for arbitrary shift $p \in \mathbb{Z}$. Finally, since the Kerr nonlinearity is real, all the terms appear in complex conjugated pairs in $\mathcal{H}_{1}^{G}$, e.g., for every $\phi_{n, a}^{*} \phi_{m, b}^{*} \phi_{k, c} \phi_{l, d}$ there is a conjugated term $\phi_{k, c}^{*} \phi_{l, d}^{*} \phi_{n, a} \phi_{m, b}$.

Note that it is straightforward to generalize the above to two-dimensional or three-dimensional lattices by replacing the unit-cell indices $n, m$ by two- or three-component vectors with integer components, respectively, in Eq. (13).

\section{A. Necessary and sufficient condition for nonlinear caging}

Nonlinear caging is defined as the confinement of any spatially compact initial excitation. This implies-similarly to the linear case- that strictly zero unit cells remain zero indefinitely in time, preventing compact excitations from spreading and forbidding distinct compact excitations to merge. The simplest example is for the nonzero wave-function amplitudes $\psi_{n}$ to be confined over a simply connected interval

$$
\begin{array}{ll}
\psi_{n}(t) \neq 0, & |n| \leqslant M, \quad \forall t>0 . \\
\psi_{n}(t)=0, & |n|>M,
\end{array}
$$

There are also more complicated cases with multiple disconnected intervals. For convenience, we refer to the set of nonzero amplitudes as an excitation. by

The time evolution of the amplitude on site $n, a$ is governed

$$
i \dot{\phi}_{n, a}=E_{a} \phi_{n, a}+\sum_{m b ; k c, l d} V_{n a, m b ; k c, l d} \phi_{m, b}^{*} \phi_{k, c} \phi_{l, d} .
$$

Nonlinear caging implies that $\phi_{n, a}(t)=0$ for all times $t$ at site $n, a$ that had a zero amplitude $\phi_{n, a}(t=0)=0$ at initial time $t=0$. This can happen if and only if the total nonlinear contribution on the right-hand side of Eq. (15) vanishes at all times. Excluding accidental cancellations of the timedependent right-hand side terms, the caging requirement is equivalent to enforcing the vanishing of each individual term in the above sum [46]. This cancellation occurs iff unit-cell indices $n, m, k, l$ appear in pairs in all the terms of $\mathcal{H}_{1}^{G}(13)$. The sufficiency of this condition is obvious by integrating Eq. (15) with respect to $\phi_{n, a}$ and assuming all the other amplitudes are known functions of time: a zero initial condition implies $\phi_{n, a}(t>0) \equiv 0$. The necessity is proved by reductio ad impossibile: let us assume that $\phi_{n, a}(t \geqslant$ $0) \equiv 0$ and there is a pair of complex-conjugated terms in $\mathcal{H}_{1}^{G}$ (13) (as was discussed above, all the terms come in complex-conjugated pairs) that have a unit-cell index $n_{1}$ different from any other indices in the same term $m_{2}, m_{3}, m_{4}$. Then we take an initial excitation containing the sites $\left(m_{2}, b_{2}\right),\left(m_{3}, b_{3}\right),\left(m_{4}, b_{4}\right)$, where $b_{1}, b_{2}, b_{3}$ are arbitrary sites in the respective unit cells, but excluding sites in the cell $n_{1}$, e.g., $\phi_{n_{1}, a_{1}}(t=0)=0$. This guarantees at least one nonzero term on the right-hand side of Eq. (15) for the site $n_{1}, a_{1}$ inside the unit cell $n_{1}$. Therefore, $\phi_{n_{1}, a_{1}}(t>0) \neq 0$ upon integration of the equation. This contradicts our initial assumption of the zero amplitude at all times at sites $\left(n_{1}, a_{1}\right)$.

Therefore, all the unit cells in the amplitudes $\phi$ of the interaction terms in Eq. (13) have to appear in pairs, so that Eq. (15) becomes

$$
\begin{aligned}
i \dot{\phi}_{n, a}= & E_{a} \phi_{n, a}+\sum_{b ; m c, d} V_{n a, n b ; m c, m d} \phi_{n, b}^{*} \phi_{m, c} \phi_{m, d} \\
& +\sum_{b ; m c, d} V_{n a, m b ; n c, m d} \phi_{m, b}^{*} \phi_{n, c} \phi_{m, d} \\
\phi_{n, a}(t=0)= & 0 .
\end{aligned}
$$

The corresponding Hamiltonian becomes

$$
\begin{aligned}
\mathcal{H}_{1}^{G}= & \sum_{n a, b ; m c, d} V_{n a, n b ; m c, m d} \phi_{n, a}^{*} \phi_{n, b}^{*} \phi_{m, c} \phi_{m, d} \\
& +\sum_{n a, b ; m c, d} V_{n a, m b ; n c, m d} \phi_{n, a}^{*} \phi_{m, b}^{*} \phi_{n, c} \phi_{m, d}
\end{aligned}
$$

To achieve caging, it is necessary and sufficient if a given Hamiltonian with a linear ABF part can be transformed into Eq. (17) in the detangled basis.

Our approach can be readily extended to more complicated interactions, e.g., involving higher powers of densities or nonlocal interactions (as has been pointed out already in Ref. [23]), as well as to higher lattice dimensions, whenever a single-particle Hamiltonian admits detangling [34], which we conjecture to be true for any ABF Hamiltonian.

Let us also note that the above proof of the caging conditions applies even to multiband dispersive lattices with fine-tuned nonlinearities that take the form (17) in the space of normal modes, e.g., where $n, a$ labels normal modes of the linear part of the Hamiltonian rather than sites.

We arrived at a direct way to test for caging in a given nonlinear AFB network with local nonlinearity: transform into the detangled basis, obtain the transformed interaction Hamiltonian, and check that all the nonlinear terms are included in Eq. (17). If they are, the nonlinear network exhibits caging, otherwise it does not.

Now we can address the question of whether a given linear $\mathrm{ABF}$ manifold contains a submanifold that supports nonlinear caging when adding local Kerr-like nonlinearities. We remind the reader that the $\mathrm{ABF}$ manifold is supposed to contain the detangled ABF Hamiltonian member. The manifold members are connected by the action of a pair of unitary transformations. Each unitary transformation is controlled by $v^{2}$ parameters. The nonlinear caging reduces to zeroing a number of dangerous terms in the transformed nonlinearity, 

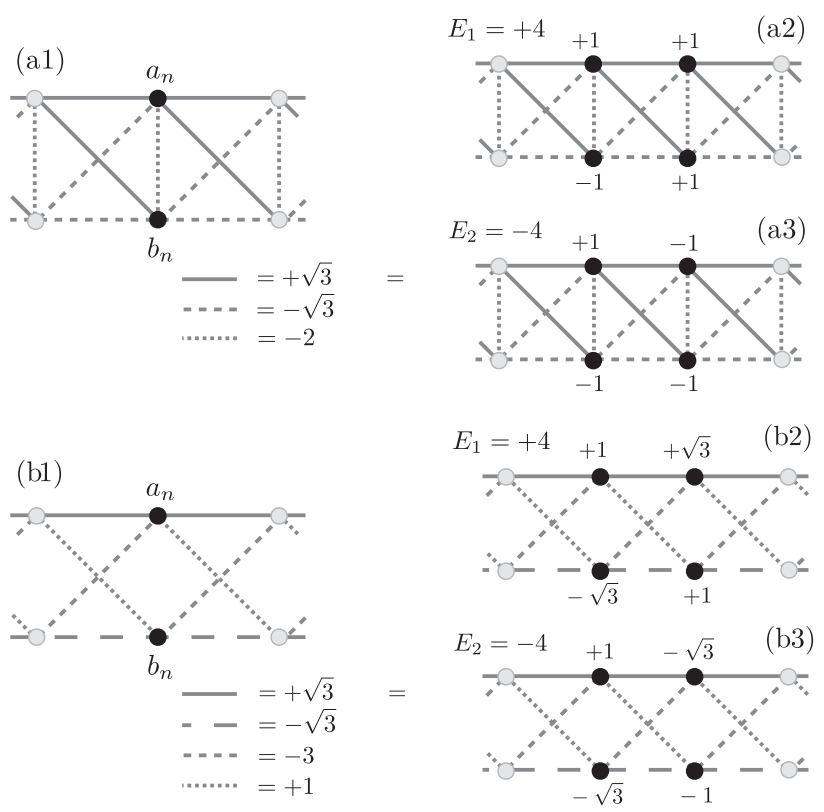

FIG. 2. (a1) Schematic representation of model A, Eq. (22). (a2),(a3) CLSs of the two flatbands at $E_{1,2}= \pm 4$. (b1) Schematic representation of model B, Eq. (23). (b2),(b3) CLSs of the two flatbands at $E_{1,2}= \pm 4$.

which amounts to the same number of equations for the unitary transformation parameters. Let us zero one element in the unitary transformation $U_{1}$. That leads to a zeroing of $v^{3}$ nonlinear coefficients $V$ in Eq. (13). It therefore appears that we can always remove all noncaging terms and remain with a nonempty submanifold of nonlinear caging Hamiltonians.

\section{B. Two-band networks}

We now illustrate the above generic result for nonlinear caging with examples drawn from the fully parametrized class of $v=2$ ABF networks (5) and (6). Equation (7) with $\psi_{n}=$ $\left(a_{n}, b_{n}\right)$ results in

$$
i \dot{\psi}_{n}=-H_{0} \psi_{n}-H_{1} \psi_{n+1}-H_{1}^{\dagger} \psi_{n-1}+U F\left(\left|\psi_{n}\right|^{2}\right) \psi_{n},
$$

with $F\left(\left|\psi_{n}\right|^{2}\right)=\left(\begin{array}{cc}\left|a_{n}\right|^{2} & 0 \\ 0 & \left|b_{n}\right|^{2}\end{array}\right)$,

while the nonlinear Hamiltonian $\mathcal{H}_{1}^{G}$ reads

$$
\mathcal{H}_{1}^{G}=\frac{U}{2} \sum_{n \in \mathbb{Z}}\left[\left|a_{n}\right|^{4}+\left|b_{n}\right|^{4}\right] .
$$

The caging condition can be established already in the $p_{n}, f_{n}$ representation (see Fig. 1). Indeed, the transformation $U_{2}$ (see Fig. 3) only affects the couplings inside one unit cell and therefore cannot introduce terms that violate the nonlinear caging criterion. This results in the following necessary and sufficient condition (see Appendix B 1 for details):

$$
\left|w_{1}\right|^{2}=\left|z_{1}\right|^{2}
$$

Then the Hamiltonian $\mathcal{H}_{1}^{G}$ in Eq. (10) recast via the transformations $U_{1}$ and $T$ in Eqs. (3) and (4) is represented by the
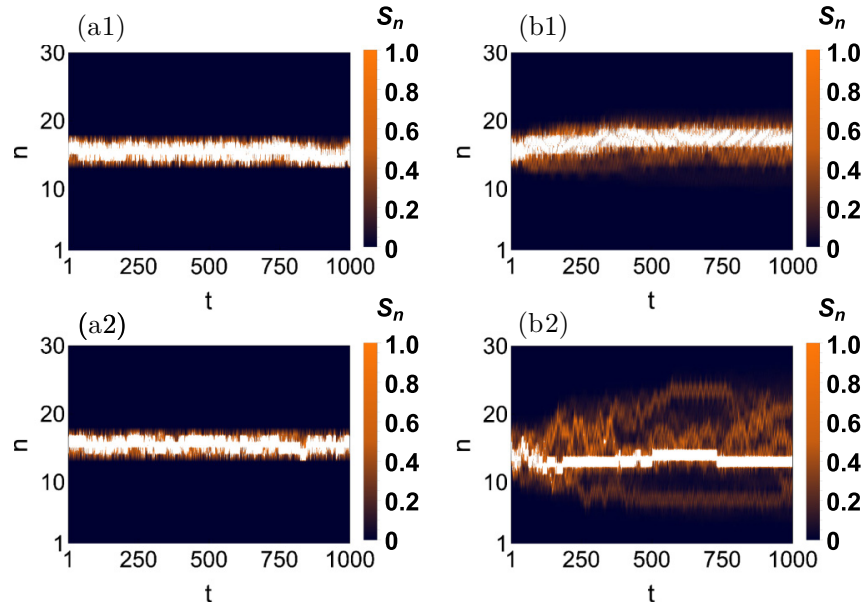

FIG. 3. (a1),(a2) Time evolution of $\mathrm{IC}_{1}$ according to model A, Eq. (22), for $U=1$ and 5, respectively. (b1),(b2) same as (a1),(a2) for model B, Eq. (23).

amplitudes $\left(p_{n}, f_{n}\right)$ (see Appendix B 2),

$$
\begin{aligned}
\mathcal{H}_{1}^{G}= & U \sum_{n}\left\{\left|z_{1}\right|^{4}\left[\left|p_{n}\right|^{4}+\left|f_{n}\right|^{4}+4\left|p_{n}\right|^{2}\left|f_{n+1}\right|^{2}\right]\right. \\
& \left.+z_{1}^{* 2} w_{1}^{2} p_{n}^{* 2} f_{n+1}^{2}+z_{1} w_{1}^{* 2} p_{n}^{2} f_{n+1}^{* 2}\right\} .
\end{aligned}
$$

The full Hamiltonian in the detangled representation is obtained in Appendix B 3 for two cases-one that satisfies caging, and another that does not. The condition (20) leads to a fine-tuned subclass of nonlinear lattices that support nonlinear caging.

\section{Two examples}

We test two example networks parametrized via Eqs. (5) and (6) - one that satisfies Eq. (20) and one that does notgenerated by setting $z_{i}=\cos \varphi_{i}, w_{i}=\sin \varphi_{i}$ in Eqs. (5) and (6).

Model $A$ is obtained by setting $\varphi_{1}=\pi / 4$ and $\varphi_{2}=\pi / 6$ and it satisfies nonlinear caging Eq. (20):

$$
\begin{aligned}
& i \dot{a}_{n}=-2 b_{n}+\sqrt{3}\left(a_{n+1}+a_{n-1}+b_{n+1}-b_{n-1}\right)+U a_{n}\left|a_{n}\right|^{2}, \\
& i \dot{b}_{n}=-2 a_{n}-\sqrt{3}\left(b_{n+1}+b_{n-1}+a_{n+1}-a_{n-1}\right)+U b_{n}\left|b_{n}\right|^{2} .
\end{aligned}
$$

The network schematics is shown in Fig. 2(a1). For $U=0$, there are two flatbands at $E_{1,2}= \pm 4$ with the respective CLSs shown in Figs. 2(a2) and 2(a3). Notably, model A cannot be obtained from the previously studied Creutz ladder nor from its gauge transformation related partners since $\varphi_{1} \neq \varphi_{2}$.

Model $B$ is obtained by setting $\varphi_{1}=\pi / 6$ and $\varphi_{2}=\pi / 4$. This model does not provide nonlinear caging since the finetuning condition (20) is violated. The equations read

$$
\begin{aligned}
& i \dot{a}_{n}=\sqrt{3} a_{n+1}+\sqrt{3} a_{n-1}+b_{n+1}-3 b_{n-1}+U a_{n}\left|a_{n}\right|^{2}, \\
& i \dot{b}_{n}=-\sqrt{3} b_{n+1}-\sqrt{3} b_{n-1}-3 a_{n+1}+a_{n-1}+U b_{n}\left|b_{n}\right|^{2} .
\end{aligned}
$$

The network schematics is shown in Fig. 2(b1). For $U=0$, there are two flatbands at $E_{1,2}= \pm 4$ with the respective CLSs shown in Figs. 2(b2) and 2(b3). 


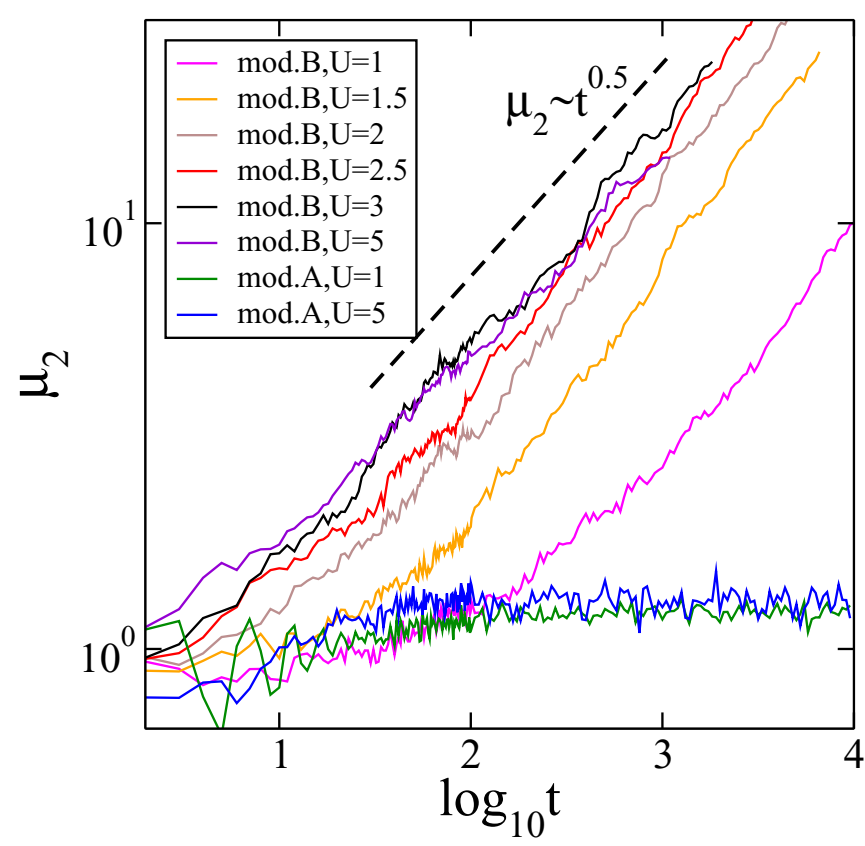

FIG. 4. Time evolution of the second moment $\mu_{2}$ over an ensemble of 48 initial conditions according to model B, Eq. (23) (upper five curves) and model A, Eq. (22) (bottom two curves) with $N=40$ for different $U$.

We visualize the presence (absence) of nonlinear caging in these models by numerically computing the time evolution of an initially compact localized excitation. We use second-order splitting $\mathrm{ABC}$ schemes for the numerical integration [47]. We consider a sample compact excitation $\mathrm{IC}_{1}$ spanning over two unit cells, and we evolve the local density $S_{n}=\left|a_{n}\right|^{2}+\left|b_{n}\right|^{2}$. The results are shown in Fig. 3 for both models A [Eq. (22)] and B [Eq. (23)] and for two interaction strengths $U=1$ and 5. For model A, Eq. (22) - panels (a1) and (a2) - the initial compact excitation $\mathrm{IC}_{1}$ remains confined within four unit cells, confirming the expected nonlinear caging. For model B, Eq. (23) - panels (b1) and (b2) - the initial excitation is propagating into the chain, confirming that caging is lost.

In Fig. 4, we show the time evolution of the second moment $\mu_{2}$ defined as

$$
\mu_{2}=\sum_{n=1}^{N}\left[(X-n)^{2}\left(\left|a_{n}\right|^{2}+\left|b_{n}\right|^{2}\right)\right]
$$

with $X=\sum_{n=1}^{N}\left[n\left(\left|a_{n}\right|^{2}+\left|b_{n}\right|^{2}\right)\right]$ for both models. The curves have been averaged over an ensemble of 48 compact initial conditions spanning over two unit cells all chosen with the same total norm $S=\sum_{n} S_{n}=7$. In the case of the cagepreserving model A, Eq. (22), we observe no signature of spreading as the second moment remains $\mu_{2} \sim 1$ over time. In the case of the noncaging preserving model B, Eq. (23), we observe a subdiffusive spreading regime: within the studied time-window, our data agree semiquantitatively with $\mu_{2} \sim$ $t^{0.5}$ for various values of the interaction strength $U$. The details of this process and its relation to previous studies of nonlinear destruction of Anderson localization [6] are certainly an interesting future project. We conjecture here that subdiffusion results from weak interactions renormalizing the compact
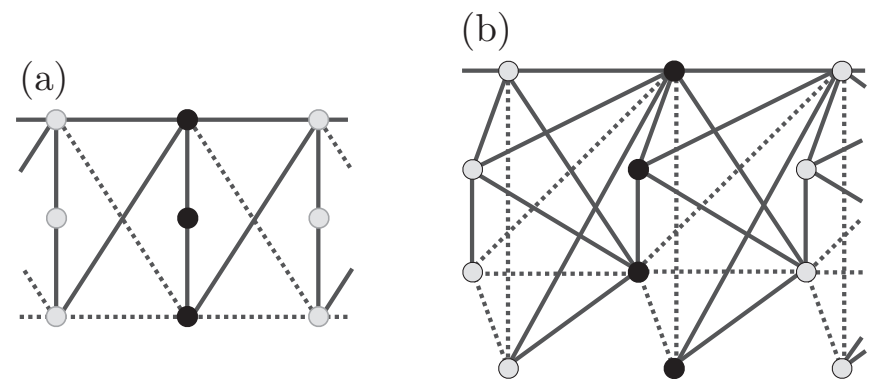

FIG. 5. Sample $v=3$ (a) and $v=4$ (b) ABF lattices defined in Eqs. (25) and (27), respectively. For the sake of simplicity, in these drawings all parameters $\mu, \chi$ have been set to 0 . Solid lines: hopping amplitude $t=+1$. Dashed lines; hopping amplitude $t=-1$. These lattices preserve nonlinear caging.

localized states and inducing nonlinear interactions between them. Both effects are proportional to the local norm density, which decreases with further spreading of the wave packet.

\section{Generalizations to more bands and higher lattice dimensions}

We now make use of the approach introduced in Sec. III A and employed in Sec. III B for one-dimensional $v=2$ lattices to present examples of cage-preserving nonlinear lattices with $v \geqslant 3$ and higher lattice dimensions.

In one dimension, we consider the family of $v=3 \mathrm{ABF}$ lattices Eq. (7) defined by

$$
H_{0}=\left(\begin{array}{ccc}
\mu & 1 & -\mu \\
1 & 0 & 1 \\
-\mu & 1 & \mu
\end{array}\right), \quad H_{1}=\left(\begin{array}{ccc}
1 & \chi & 1 \\
0 & 0 & 0 \\
-1 & -\chi & -1
\end{array}\right)
$$

with free parameters $\mu, \chi$. The family-shown in Fig. 5(a) for $\mu, \chi=0$ for clarity-preserves nonlinear caging since the unitary transformation that detangles the linear part,

$$
U_{\nu=3}=\frac{1}{\sqrt{2}}\left(\begin{array}{ccc}
1 & 0 & 1 \\
0 & \sqrt{2} & 0 \\
-1 & 0 & 1
\end{array}\right)
$$

does not generate any forbidden transporting nonlinear terms in the detangled representation. The $v=3 \mathrm{ABF}$ family in Eq. (25) is a submanifold of the full $v=3 \mathrm{ABF}$ manifold. The full manifold also contains the ABF diamond chain, which preserves nonlinear caging as well as that studied in Refs. [22,23], but it is not part of the above example family. This demonstrates that previously observed ABF networks which satisfy nonlinear caging are single members of entire families of multiparameter $\mathrm{ABF}$ submanifolds that preserve nonlinear caging.

The very same reasoning applies to larger numbers of bands, e.g., for $v=4$. The family of nonlinear lattices Eq. (7) defined by

$$
H_{0}=\left(\begin{array}{cccc}
\mu_{1} & 1 & -\mu_{1} & -1 \\
1 & \mu_{2} & 1 & \mu_{2} \\
-\mu_{1} & 1 & \mu_{1} & -1 \\
-1 & \mu_{2} & -1 & \mu_{2}
\end{array}\right)
$$



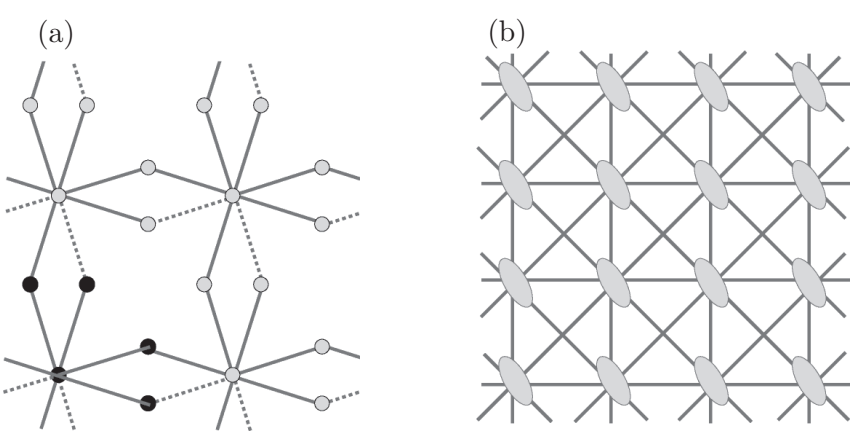

FIG. 6. Nonlinear caging in two dimensions. (a) $v=5$ cagepreserving two-dimensional (decorated Lieb) lattice. Solid lines indicate hopping +1 , dashed lines indicate hopping -1 . (b) $v=$ 2 cage-preserving two-dimensional lattice with second-nearestneighbor hopping defined in Eqs. (29) and (30). For the sake of clarity, the tilted ellipses represent the unit cells, while the solid lines represent the hopping matrix elements Eq. (30).

$$
H_{1}=\left(\begin{array}{cccc}
1 & \chi_{1} & 1 & -\chi_{1} \\
1 & \chi_{2} & 1 & -\chi_{2} \\
-1 & -\chi_{1} & -1 & \chi_{1} \\
1 & \chi_{2} & 1 & -\chi_{2}
\end{array}\right)
$$

with free parameters $\mu_{1}, \mu_{2}, \chi_{1}, \chi_{2}$-shown in Fig. 5(b) with $\mu_{1}, \mu_{2}, \chi_{1}, \chi_{2}=0$ for clarity-is cage-preserving. Indeed, the unitary transformation that detangles the linear part

$$
U_{\nu=4}=\frac{1}{\sqrt{2}}\left(\begin{array}{cccc}
1 & 0 & 1 & 0 \\
0 & 1 & 0 & 1 \\
-1 & 0 & 1 & 0 \\
0 & -1 & 0 & 1
\end{array}\right)
$$

does not introduce any dangerous transporting nonlinear terms in the detangled representation.

In two dimensions, we use the $v=5$ two-dimensional lattice called the decorated Lieb lattice [48] as one example that satisfies nonlinear caging [see Fig. 6(a)].

Another example is a novel two-dimensional $v=2 \mathrm{ABF}$ lattice with additional diagonal hopping connectivities [see Fig. 6(b)]:

$$
\begin{aligned}
i \dot{\psi}_{n, m}= & -H_{1, n} \psi_{n+1, m}-H_{1, n}^{\dagger} \psi_{n-1, m} \\
& -H_{1, m} \psi_{n, m+1}-H_{1, m}^{\dagger} \psi_{n, m-1} \\
& -H_{2, D} \psi_{n+1, m+1}-H_{2, D}^{\dagger} \psi_{n-1, m-1} \\
& -H_{2, A} \psi_{n+1, m-1}-H_{2, A}^{\dagger} \psi_{n-1, m+1} \\
& +U F\left(\left|\psi_{n, m}\right|^{2}\right) \psi_{n, m}
\end{aligned}
$$

with

$$
\begin{array}{ll}
H_{1, n}=-\frac{\sqrt{3}}{4}\left(\begin{array}{cc}
0 & 1 \\
1 & 0
\end{array}\right), & H_{1, m}=\frac{1}{4}\left(\begin{array}{cc}
-1 & -1 \\
1 & 1
\end{array}\right), \\
H_{2, D}=\frac{\sqrt{3}}{8}\left(\begin{array}{cc}
1 & 1 \\
-1 & -1
\end{array}\right), & H_{2, A}=\frac{\sqrt{3}}{8}\left(\begin{array}{ll}
-1 & 1 \\
-1 & 1
\end{array}\right) .
\end{array}
$$

This model is cage-preserving since the detangling process obtained by the unitary transformation,

$$
U_{\nu=2}=\frac{1}{\sqrt{2}}\left(\begin{array}{cc}
1 & 1 \\
-1 & 1
\end{array}\right)
$$

twice alternated by the redefinition $\psi_{n, m} \longmapsto \psi_{n, m-1}$, reduces the Kerr nonlinearity to the form given by Eq. (17).

We remark that certain models presented above-model A [Eq. (22)], the $v=31 \mathrm{D}$ model [Eq. (25)], and the decorated Lieb lattice in Fig. 6(a)-possess local permutation symmetries [30] implying the existence of local conserved quantities in the quantum case $[30,32]$.

\section{DISCUSSIONS AND PERSPECTIVES}

In this work, we showed that in one dimension, dispersionless networks can be completely detangled via local unitary transformations. The inversion of that procedure yields a systematic generator for all band flat networks with finite-range hopping terms in any lattice dimension [34]. We then studied the impact of classical nonlinear interactions on lattices without linear dispersion and formulated necessary and sufficient conditions for nonlinear caging. We used two-band networks as testbeds to show that single-particle caging is in general broken by classical interactions. We further extended our analysis to three- and four-band networks, and we went into two dimensions with two- and five-band models, respectively, which again show the possibility of nonlinear caging.

An observation aligned with Ref. [23], which follows from the detangling procedure developed in this work, is that nonlinear caging is not specific to Kerr nonlinearity but holds also for other, even nonlocal nonlinear terms. The detangling method leads to a broad number of nontrivial $\mathrm{ABF}$ caged models with complicated nonlocal nonlinear interaction terms. Interesting future challenges include the search for experimentally feasible examples, and their potentially novel features beyond the known ABF models with local Kerr nonlinearity. Another thrilling question we intend to address in a related work concerns the interacting quantum many-body dynamics in ABF networks which satisfy nonlinear caging.

\section{ACKNOWLEDGMENTS}

The authors thank Ihor Vakulchyk, Ajith Ramachandran, Arindam Mallick, and Tilen Čadez for helpful discussions. This work was supported by the Institute for Basic Science, Korea (IBS-R024-D1).

\section{APPENDIX A: DETANGLING OF ABF ONE-DIMENSIONAL NETWORKS}

In this Appendix, we provide the proof of the Theorem in Sec. II stating that any $d=1$ Hamiltonian with all bands flat and finite-range hopping can be recast in a set of decoupled sites via a sequence of local unitary transformation. That is equivalent to a statement that in these Hamiltonians all compact localized states are orthogonal and can be recast to have nonzero amplitude over a single unit cell.

Throughout this Appendix, we will use $\langle A, B\rangle$ to denote the scalar product of matrices: $\langle A, B\rangle=\operatorname{Tr}\left(A^{\dagger} B\right)$ or vectors $\langle A, B\rangle=\sum_{a} A_{a}^{*} B_{a}$, and we will denote with $u$ the number of unit cells occupied by the CLS. 


\section{Preliminaries}

We consider a 1D lattice Hamiltonian with $v$ bands, of which one or more are flat. The Hamiltonian is parametrized by intra-unit-cell hopping matrix $H_{0}$ and inter-unit-cell hopping matrices $H_{m}, m>0[40,43]$. We assume that $H_{m}$ describes hopping to the left, while $H_{m}^{\dagger}$ describes the hopping to the right from a given unit cell. For simplicity, we consider the case of nearest-neighbor hopping. Then the Bloch Hamiltonian reads

$$
\mathcal{H}_{q}=e^{i q} H_{1}^{\dagger}+H_{0}+e^{-i q} H_{1} .
$$

First we define the tensor $C_{\alpha, a b}$ that parametrizes a CLS of size $u$ of a flatband with energy $\varepsilon_{\alpha}$ in a 1D model with $v$ bands: $\alpha$ is a band index, while $b$ indicates the unit cells and runs from 1 to $u$, and $a$ is the site index in the unit cell $b$. The $v \times u$ matrix $C_{\alpha}$ is parametrizing the CLS of band $\alpha$, and it is the central object of the proof presented below.

For concreteness, we provide a well-studied example of an ABF system - the diamond chain in a magnetic field at half-flux studied in the context of nonlinear caging [22-24]. We refer to this model simply as the diamond chain in this Appendix. In the case of the diamond chain, the three tensors $C_{\alpha, a b}$ read (see Fig. 2 of Ref. [24])

$$
C_{1}=\left(\begin{array}{cc}
1 & -1 \\
0 & 0 \\
1 & 1
\end{array}\right), \quad C_{2}=\left(\begin{array}{cc}
1 & 1 \\
0 & -2 \\
1 & -1
\end{array}\right), \quad C_{3}=\left(\begin{array}{cc}
1 & 1 \\
0 & 2 \\
1 & -1
\end{array}\right)
$$

Next we discuss the correspondence between the CLS tensor $C_{\alpha, a b}$ and the FB Bloch eigenfunction: knowing a CLS, we can always construct a normalized Bloch eigenfunction:

$$
\begin{aligned}
\Psi_{\alpha, a}(q) & =\frac{\sum_{b=0}^{u-1} C_{\alpha, a b} \omega_{q b}}{\sqrt{\left\langle C_{\alpha} \omega_{q}, C_{\alpha} \omega_{q}\right\rangle}}, \\
\omega_{q} & =\left(1, e^{i q}, \ldots, e^{i q(u-1)}\right) .
\end{aligned}
$$

To prove that this is an eigenfunction, we need to verify $\mathcal{H}_{q} \Psi_{\alpha, a}(q)=\varepsilon_{\alpha} \Psi_{\alpha, a}(q)$ : using the Hamiltonian (A1) and plugging the above expression for $\Psi$ and vanishing the coefficients in front of $e^{i q a}$, we find

$$
\begin{gathered}
H_{0} \vec{\psi}_{\alpha, 0}+H_{1} \vec{\psi}_{\alpha, 1}=\varepsilon_{\alpha} \vec{\psi}_{\alpha, 0}, \\
H_{1}^{\dagger} \vec{\psi}_{\alpha, a-1}+H_{0} \vec{\psi}_{\alpha, a}+H_{1} \vec{\psi}_{\alpha, a+1}=\varepsilon_{\alpha} \vec{\psi}_{\alpha, a}, \\
a=1, \ldots, u-2, \\
H_{1}^{\dagger} \vec{\psi}_{\alpha, u-2}+H_{0} \vec{\psi}_{\alpha, u-1}=\varepsilon_{\alpha} \vec{\psi}_{\alpha, u-1}, \\
H_{1} \vec{\psi}_{\alpha, 0}=H_{1}^{\dagger} \psi_{\alpha, u-1}=0, \\
\vec{\psi}_{\alpha, b}=\left(C_{\alpha, 1 b}, \ldots, C_{\alpha, v b}\right) .
\end{gathered}
$$

This is precisely a system of eigenvalue equations with destructive interference conditions satisfied by a CLS of size $u$ in a 1D system with nearest-neighbor hopping [43]. We note that the inverse also holds - if an eigenfunction of a 1D FB takes the form (A3), it immediately reconstructs the CLS tensor $C_{\alpha, a b}$ from the expansion of the eigenfunction in powers of $e^{i q}$. The highest power of $e^{i q}$ defines the size of the CLS. Based on this correspondence, we conclude that a $q$-independent Bloch wave function corresponds to the $U=1$ CLS.

Finally, as a warmup for the proof, we demonstrate how the diamond chain Hamiltonian is diagonalized by a local unitary transformation and a unit-cell redefinition. We apply the unitary transformation to the original wave functions $\psi_{n}=$ $\left(\psi_{n, 1}, \psi_{n, 2}, \psi_{n, 2}\right)$ :

$$
\varphi_{n}=\left(\begin{array}{c}
\varphi_{n, 1} \\
\varphi_{n, 2} \\
\varphi_{n, 3}
\end{array}\right)=U_{1}^{\dagger}\left(\begin{array}{l}
\psi_{n, 1} \\
\psi_{n, 2} \\
\psi_{n, 3}
\end{array}\right), \quad U_{1}=\frac{1}{\sqrt{2}}\left(\begin{array}{ccc}
1 & -1 & 0 \\
0 & 0 & \sqrt{2} \\
1 & 1 & 0
\end{array}\right) .
$$

The transformation is chosen to convert the columns of $C_{1}$ into basis vectors. This transforms the hopping matrices $H_{0}, H_{1}$ as follows:

$$
\begin{aligned}
& U_{1}^{\dagger} H_{0} U_{1}^{\dagger}=-\left(\begin{array}{ccc}
0 & 0 & 0 \\
0 & 0 & \sqrt{2} \\
0 & \sqrt{2} & 0
\end{array}\right), \\
& U_{1}^{\dagger} H_{1} U_{1}=\left(\begin{array}{ccc}
0 & 0 & 0 \\
0 & 0 & 0 \\
\sqrt{2} & 0 & 0
\end{array}\right) .
\end{aligned}
$$

The CLS tensors become

$$
\begin{gathered}
D_{1}=U_{1}^{\dagger} C_{1}=\sqrt{2}\left(\begin{array}{cc}
1 & 0 \\
0 & 1 \\
0 & 0
\end{array}\right), \\
D_{2}=U_{1}^{\dagger} C_{2}=\sqrt{2}\left(\begin{array}{cc}
1 & 0 \\
0 & -1 \\
0 & -\sqrt{2}
\end{array}\right), \\
D_{3}=U_{1}^{\dagger} C_{1}=\sqrt{2}\left(\begin{array}{cc}
1 & 0 \\
0 & -1 \\
0 & \sqrt{2}
\end{array}\right) .
\end{gathered}
$$

We observe that all the new tensors $D_{\alpha}=U_{1}^{\dagger} C_{\alpha}$ share the same pattern of zero elements, so that we can redefine the unit cell as follows:

$$
\phi_{n}=\left(\begin{array}{c}
\phi_{n, 1} \\
\phi_{n, 2} \\
\phi_{n, 2}
\end{array}\right)=U_{2} \varphi_{n}=\left(\begin{array}{c}
\varphi_{n-1,1} \\
\varphi_{n, 2} \\
\varphi_{n, 3}
\end{array}\right)
$$

to fit CLS in a single unit cell after the redefinition $U_{2}$. The CLS after the unit-cell redefinition read

$$
\begin{gathered}
E_{1}=U_{2}^{\dagger} D_{1}=\sqrt{2}\left(\begin{array}{ll}
1 & 0 \\
1 & 0 \\
0 & 0
\end{array}\right), \\
E_{2}=U_{2}^{\dagger} D_{2}=\sqrt{2}\left(\begin{array}{cc}
1 & 0 \\
-1 & 0 \\
-\sqrt{2} & 0
\end{array}\right), \\
E_{3}=U_{2}^{\dagger} D_{1}=\sqrt{2}\left(\begin{array}{cc}
1 & 0 \\
-1 & 0 \\
\sqrt{2} & 0
\end{array}\right) .
\end{gathered}
$$

The new CLS $E_{\alpha}$ effectively occupy a single unit cell, which is directly transformed into decoupled sites [34]: the transformation $U=U_{2} U_{1}$ reduced the original diamond chain Hamiltonian to an ABF system with CLS of size $u=1$. In this case, the same conclusion follows from a direct inspection of 
the transformed model with updated hopping matrices (A10): the chain splits into disconnected clusters that can be fit into a single new unit cell.

The generic proof presented below shows that the above result for the ABF diamond chain is a generic feature of 1D ABF models with short-range (compact) hopping: there always exists a finite sequence of unitary transformations, like $U_{1}$, and unit-cell redefinitions, like $U_{2}$, that reduce these models to decoupled sites. Unlike the diamond chain, the reduction might require several applications of the analogs of the transformation $U_{2} U_{1}$ discussed above, before the decoupling of the sites is achieved. This is achieved by considering an arbitrary $\mathrm{ABF}$ Hamiltonian taking into account the constraints on the CLS tensors $C_{\alpha}$ : orthogonality of the eigenstates and the shortrange hopping in the Hamiltonian.

For the convenience of the reader, we keep in the proof below the same notation, $\psi_{n}, \varphi_{n}, \phi_{n}$ for the wave-function amplitudes and $C_{\alpha}, D_{\alpha}, E_{\alpha}$ for the CLS amplitudes in the original basis, after the basis redefinition and unit-cell change, respectively.

\section{Proof}

We consider a $d=1$ Hamiltonian with $v$ bands that are all flat. From Ref. [33] it follows that all of its eigenstates can be represented as spatially compact, e.g., as CLS. We assume that the largest CLS has size $u$ (in the case of CLS of different size, we assume that they are all padded by zeros from the right to the size of the largest CLS). If flatbands with CLS of size $u=1$ are present in the spectrum, we can apply the detangling procedure of Ref. [34] and use local unitary transformations to turn these FBs into completely decoupled sites, while the rest of the Hamiltonian keeps all bands flat and has short-range hopping. Therefore, without loss of generality we assume that there are no flatbands with CLS of size $u=1$.

\section{a. Orthogonality conditions}

The CLS are encoded by their tensors $C_{\alpha, a b}$ of size $v \times$ $v \times u$. Therefore, as was discussed above, we can express the Bloch eigenfunctions of the Hamiltonian as

$$
\begin{aligned}
\Psi_{\alpha, a}(q) & =\frac{\sum_{b=0}^{u-1} C_{\alpha, a b} \omega_{q b}}{\sqrt{\left\langle C_{\alpha} \vec{\omega}_{q}, C_{\alpha} \vec{\omega}_{q}\right\rangle}} \propto \sum_{b=0}^{u-1} C_{\alpha, a b} e^{i b q}, \\
\vec{\omega}_{q} & =\left(1, e^{i q}, \ldots, e^{i q(u-1)}\right),
\end{aligned}
$$

where $\alpha$ is the band index, and $a$ is the wave-function component. The eigenstates of a Hermitian Hamiltonian have to be orthogonal, giving the first set of constraints on the matrices $C_{\alpha}$ :

$$
\begin{aligned}
\delta_{\alpha \beta} & =\sum_{a=1}^{\nu} \Psi_{\alpha, a}^{*}(q) \Psi_{\beta, a}(q) \\
& \propto \sum_{a b c} e^{i q(c-b)} C_{\alpha, a b}^{*} C_{\beta, a c}=\sum_{b c} e^{i q(c-b)} T_{\alpha \beta, b c}, \\
T_{\alpha \beta, a b} & =\sum_{c=1}^{\nu} C_{\alpha, c a}^{*} C_{\beta, c b} .
\end{aligned}
$$

The tensors $T$ have size $v \times v \times u \times u$. The above orthogonality condition reduces to a special Fourier transform of the tensors $T_{\alpha \beta}$, which implies that the tensors $T_{\alpha \beta}, \alpha \neq \beta$ should have zero sum of elements over any diagonal. In particular,

$$
T_{\alpha \beta, 1 u}=T_{\alpha \beta, u 1}=0, \quad \alpha \neq \beta .
$$

Using the parametrization of the eigenstates (A15) and taking the flatband energies as $\varepsilon_{\alpha}$, we write the Hamiltonian using the spectral decomposition:

$$
\begin{aligned}
\mathcal{H}_{q} & =M_{q} \Lambda M_{q}^{\dagger}, \quad \Lambda_{a b}=\varepsilon_{a} \delta_{a b}, \\
M_{q} & =\left(\vec{\Psi}_{1} \vec{\Psi}_{2}, \ldots, \vec{\Psi}_{\nu}\right), \\
\vec{\Psi}_{\alpha} & =\frac{C_{\alpha} \vec{\omega}_{q}}{\sqrt{\left\langle C_{\alpha} \vec{\omega}_{q}, C_{\alpha} \vec{\omega}_{q}\right\rangle}} .
\end{aligned}
$$

The Hamiltonian becomes

$$
\begin{aligned}
\mathcal{H}_{q}= & \sum_{\alpha=1}^{\nu} \varepsilon_{\alpha} \vec{\Psi}_{\alpha} \otimes \vec{\Psi}_{\alpha}^{*}=\sum_{\alpha} \varepsilon_{\alpha} \frac{P_{\alpha}\left(e^{i q}\right)}{Q_{\alpha}\left(e^{i q}\right)}, \\
P_{\alpha}\left(e^{i q}\right)=\left(C_{\alpha} \vec{\omega}_{q}\right) \otimes\left(C_{\alpha} \vec{\omega}_{q}\right)^{*} & \\
Q_{\alpha}\left(e^{i q}\right)= & \left\langle C_{\alpha} \vec{\omega}_{q}, C_{\alpha} \vec{\omega}_{q}\right\rangle \\
= & \sum_{b, c=1}^{\nu} C_{\alpha, b c}^{*} C_{\alpha, b c} e^{i q(b-c)}=\sum_{b c=1}^{\nu} e^{i q(b-c)} T_{\alpha \alpha, b c} .
\end{aligned}
$$

Here, $P_{\alpha}(z)$ is a Laurent $v \times v$ matrix polynomial in powers of $z=e^{i q}$, while $Q_{\alpha}(z)$ is a Laurent polynomial in powers of $z=$ $e^{i q}$. The size of the CLS $u$ and Eq. (A15) impose the following constraint on the degrees of both Laurent polynomials:

$$
\begin{aligned}
\operatorname{deg} P_{\alpha, a b}\left(e^{i q}\right) \leqslant u-1, \quad \forall a, b, \\
\operatorname{deg} Q_{\alpha}\left(e^{i q}\right) \leqslant u-1 .
\end{aligned}
$$

The Hamiltonian is a weighted sum of the ratios of these two sets of polynomials, and every ratio $P_{\alpha}\left(e^{i q}\right) / Q_{\alpha}\left(e^{i q}\right)$ is a projector on the respective eigenvector $\vec{\Psi}_{\alpha}(q)$.

Without further constraints, the above Hamiltonian is longranged. Indeed, for generic CLS tensors $C_{\alpha}$ satisfying (A16), $P_{\alpha, a b}(q)$ is not exactly divisible by $Q_{\alpha}(q)$ and their ratio generates an infinite Laurent series in powers of $e^{i q}$. Therefore, the Hamiltonian $\mathcal{H}$ has hopping of arbitrary range.

The Hamiltonian becomes short-ranged iff $P_{\alpha, a b}$ is exactly divisible by $Q_{\alpha}$ for all $\alpha$ and $a, b$. If $\operatorname{deg} P_{\alpha, a b}=\operatorname{deg} Q_{\alpha}$ for all pairs $a, b$, then all the ratios $P_{\alpha, a b} / Q_{\alpha}$ are constants independent of $q$ for all $a, b$ and the respective eigenvector $\vec{\Psi}_{\alpha}(q)$ is $q$-independent, corresponding to the $u=1$ CLS. This is impossible since we assumed that the minimal size of a CLS for any band is greater than 1 . Therefore, $\operatorname{deg} P_{\alpha, a b}(q)>$ $\operatorname{deg} Q_{\alpha}(q)$ for at least one pair $a, b$ and $\operatorname{deg} Q_{\alpha}(q)<u-1$ as a consequence of Eq. (A22). Then it follows from Eq. (A21) that

$$
T_{\alpha \alpha, 1 u}=T_{\alpha \alpha, u 1}=0, \quad \forall \alpha .
$$

This condition together with constraint (A17) implies that the vector of amplitudes in the first unit cell of any CLS is orthogonal to a vector of amplitudes in the last unit cell of any CLS. 


\section{b. Unit-cell basis change $U_{1}$ and unit-cell redefinition $U_{2}$}

Constraints (A17) and (A23) are the main results of the previous part of the proof, which allow us to define the transformations $U_{1}$ (A9) and $U_{2}$ (A14) in the general case of a $1 \mathrm{D}$ $\mathrm{ABF}$ Hamiltonian with short-range hopping.

We write the CLS tensors as $C_{\alpha}=\left(\vec{c}_{\alpha 1}, \vec{c}_{\alpha 2}, \ldots, \vec{c}_{\alpha u}\right)$, where $\vec{c}_{\alpha a}$ is a column vector of the eigenfunction amplitudes in the unit cell $a$ of the CLS of the band $\alpha$. We define a basis change $U_{1}=\left(\vec{e}_{1}, \ldots, \vec{e}_{v}\right)$ of the wave-function amplitudes $\psi_{n, a}$ as a union of three sets of orthonormal vectors:

Set $\mathcal{A}$ : orthonormal vectors generated by applying GramSchmidt orthogonalization to the set $\left\{\vec{c}_{\alpha, 1}, \forall \alpha\right\}$.

Set $\mathcal{B}$ : orthonormal vectors generated by applying GramSchmidt orthogonalization to the set $\left\{\vec{c}_{\alpha, u}, \forall \alpha\right\}$.

Set $\mathcal{E}$ : if the union of sets $\mathcal{A}$ and $\mathcal{B}$ is undercomplete, then add the missing basis vectors that complete the basis, otherwise set as empty.

Because of Eqs. (A17) and (A23), all the vectors from $\mathcal{A}$ and $\mathcal{B}$ are mutually orthogonal. This ensures that the resulting set of vectors $\left\{\vec{e}_{a}\right\}$ is orthonormal and constitutes a valid new basis. The transformation $U_{1}$ is the generalization of the transformation (A9) applied to the diamond chain. The wavefunction amplitudes in the new basis $\varphi_{n, a} \operatorname{read} \varphi_{n}=U_{1}^{\dagger} \psi_{n}$. The CLS tensors transform accordingly: the tensors in the new basis $D_{\alpha}$ read $D_{\alpha}=U_{1}^{\dagger} C_{\alpha}$. Similarly to $C_{\alpha}$, we write $D_{\alpha}=\left(\vec{d}_{\alpha 1}, \vec{d}_{\alpha 2}, \ldots, \vec{d}_{\alpha u}\right)$, where $\vec{d}_{\alpha a}$ are column vectors.

Importantly, nonzero coefficients $D_{\alpha, 1 a}$ correspond to zero coefficients $D_{\beta, u a}$ and vice versa for all $\alpha, \beta$. This follows directly from Eqs. (A9) and (A14) and the definition of the basis change $U_{1}$.

Using this result, we redefine the unit cell of the network using the following unitary transformation $U_{2}$ [again generalizing the particular case of the diamond chain (A14)]:

$$
\phi_{n, a}= \begin{cases}\varphi_{n+1, a}, & D_{\alpha, 1 a}=0 \forall \alpha, \\ \varphi_{n, a} & \text { otherwise, }\end{cases}
$$

where $\phi_{n, a}$ are wave-function amplitudes in the new redefined unit cell $n$. This redefinition takes the old unit cell and constructs the new unit cell by replacing all the sites where CLS amplitudes in $\vec{d}_{\alpha, 1}$ (e.g., the first unit cell of the CLS) are zero for all bands $\alpha$ by the sites from the next unit cell.

The CLS tensors in the redefined unit cell are denoted as $E_{\alpha, a b}$ and are given by $E_{\alpha}=U_{2}^{\dagger} D_{\alpha}$ :

$$
E_{\alpha, n a}= \begin{cases}D_{\alpha, n+1, a}, & D_{\alpha, 1 a}=0 \forall \alpha, \\ D_{\alpha, n a} & \text { otherwise. }\end{cases}
$$

In this new unit cell, the last column (unit cell) of the matrix $E_{\alpha}$ is identically zero for all $\alpha$. Indeed, $D_{\alpha}$ is a matrix composed of $u$ columns, each corresponding to a unit cell. As we showed above for every nonzero amplitude $D_{\alpha, u a}$ (the last unit cell of the CLS), the amplitudes $D_{\beta, 1 a}=0$ for any $\beta$. This is precisely the condition in Eq. (A25) for the amplitude $D_{\alpha, u a}$ to be grouped together with the amplitudes from the previous unit cell, $u-1$, and to make a new redefined unit cell. Therefore, only zero amplitudes remain in the last column of every $E_{\alpha}$, and the effective size of all the CLS $E_{\alpha}$ is reduced from $u$ to $u-1$.

We end up with a new ABF Hamiltonian transformed by two unitary transformations: the introduction of a new basis
$U_{1}$ and a unit-cell redefinition $U_{2}$ (A24), but with the same spectrum as the original Hamiltonian and all the CLS sizes decreased by 1 . Repeating the entire procedure outlined above $u-2$ more times, we can reduce the sizes of all the CLS to $u=1$. This concludes the proof of the Theorem from the main text.

\section{Parametrization of $v=2$ networks}

The above proof when inverted yields a generator scheme for dispersionless networks. In this subsection, we explicitly unfold the two-band problem, $v=2$. Let us consider a nondegenerate (two different flatband energies) fully decoupled network in coordinates $\phi_{n}=\left(\alpha_{n}, \beta_{n}\right)$,

$$
\begin{gathered}
i \dot{\phi}_{n}=-H_{0}^{(1)} \phi_{n}-H_{1}^{(1)} \phi_{n+1}-H_{1}^{(1) \dagger} \phi_{n-1}, \\
H_{0}^{(1)}=\left(\begin{array}{cc}
-1 & 0 \\
0 & 1
\end{array}\right), \quad H_{1}^{(1)}=\left(\begin{array}{ll}
0 & 0 \\
0 & 0
\end{array}\right),
\end{gathered}
$$

with two flatband energies $E_{1}=-1$ and $E_{2}=1$. The mapping in Eq. (3) consists of two unitary transformations $U_{1}$ and $U_{2}$, which are parametrized as

$$
U_{i}=e^{i \theta_{i}}\left(\begin{array}{cc}
z_{i} & w_{i} \\
-w_{i}^{*} & z_{i}^{*}
\end{array}\right), \quad i=1,2
$$

by the complex numbers $z_{i}, w_{i}$ such that $\left|z_{i}\right|^{2}+\left|w_{i}\right|^{2}=1$ and the phases $\theta_{i}$. The unit-cell redefinition $T$ is

$$
T:\left\{\begin{array}{l}
p_{n} \longmapsto p_{n}, \\
f_{n} \longmapsto f_{n-1} .
\end{array}\right.
$$

We generate all the $v=2$ dispersionless lattices by applying the transformations, Eqs. (A28) and (A29), in the following
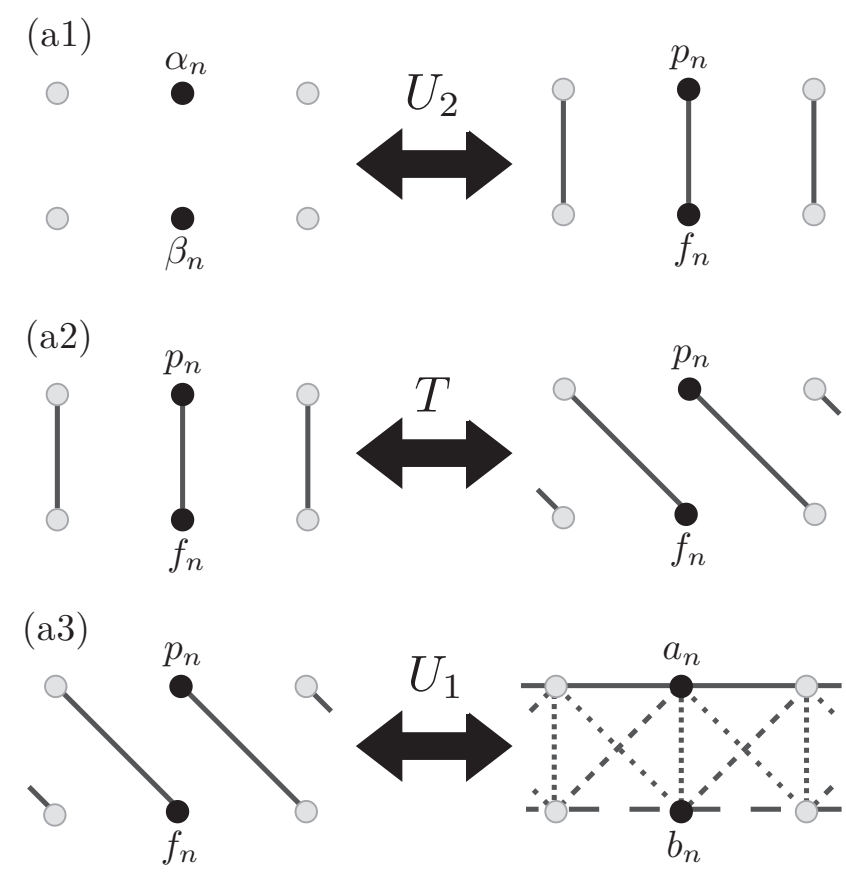

FIG. 7. Schematic representation of the unit-cell redefinition for a $v=2$-band lattice. In each panel, the black dots represent the chosen unit cell. 
order:

$$
\left(\begin{array}{c}
\alpha_{n} \\
\beta_{n}
\end{array}\right) \stackrel{U_{2}}{\longmapsto}\left(\begin{array}{c}
p_{n} \\
f_{n}
\end{array}\right) \stackrel{T}{\longmapsto}\left(\begin{array}{c}
p_{n} \\
f_{n}
\end{array}\right) \longmapsto\left(\begin{array}{l}
a_{n} \\
b_{n}
\end{array}\right),
$$

as shown in Fig. 7.

The first coordinate rotation $U_{2}$ turns $H_{0}^{(1)}$ in Eq. (A27) to

$$
H_{0}^{(2)}=U_{2} H_{0}^{(1)} U_{2}^{\dagger}=\left(\begin{array}{cc}
\left|w_{2}\right|^{2}-\left|z_{2}\right|^{2} & 2 z_{2} w_{2} \\
2 z_{2}^{*} w_{2}^{*} & \left|z_{2}\right|^{2}-\left|w_{2}\right|^{2}
\end{array}\right),
$$

while $H_{1}^{(2)}=U_{2} H_{1}^{(1)} U_{2}^{\dagger}$ remains zero-as shown in Fig. 7(a1), right plot.
The unit-cell redefinition $T$ in Eq. (A29) redefines $H_{0}^{(2)}$ and $H_{1}^{(2)}$ as

$$
\begin{gathered}
H_{0}^{(3)}=\left(\begin{array}{cc}
\left|w_{2}\right|^{2}-\left|z_{2}\right|^{2} & 0 \\
0 & \left|z_{2}\right|^{2}-\left|w_{2}\right|^{2}
\end{array}\right), \\
H_{1}^{(3)}=\left(\begin{array}{cc}
0 & 2 z_{2} w_{2} \\
0 & 0
\end{array}\right),
\end{gathered}
$$

as shown in Fig. 7(a2), right plot.

Finally, the rotation $U_{1}$ turns $H_{0}^{(3)}$ and $H_{1}^{(3)}$ in Eqs. (A32) and (A33) to the following matrices:

$$
H_{0}=U_{1} H_{0}^{(3)} U_{1}^{\dagger}=\Gamma_{0}\left(\begin{array}{cc}
\left|z_{1}\right|^{2}-\left|w_{1}\right|^{2} & -2 z_{1} w_{1} \\
-2 z_{1}^{*} w_{1}^{*} & \left|w_{1}\right|^{2}-\left|z_{1}\right|^{2}
\end{array}\right),
$$

$$
H_{1}=U_{1} H_{1}^{(3)} U_{1}^{\dagger}=\Gamma_{1}\left(\begin{array}{cc}
z_{1} w_{1}^{*} & z_{1}^{2} \\
-\left(w_{1}^{*}\right)^{2} & -z_{1} w_{1}^{*}
\end{array}\right)
$$

for $\Gamma_{0}=\left|w_{2}\right|^{2}-\left|z_{2}\right|^{2}$ and $\Gamma_{1}=2 z_{2} w_{2}$-as shown in Fig. 7(a3), right plot.

\section{APPENDIX B: DETANGLING PROCEDURE APPLIED TO NONLINEAR DISPERSIONLESS MODELS}

In this Appendix, we apply the detangling procedure as described by Eq. (3) to the dispersionless, two-band models in the presence of a local Kerr nonlinearity (10).

\section{Preserving the caging in $\boldsymbol{v}=\mathbf{2}$ networks: A necessary and sufficient condition}

We now work out the detangled local Kerr nonlinearity for a general $v=2 \mathrm{AB}$ lattice. The transformation $U_{1}$ in components reads

$$
U_{1}:\left\{\begin{array}{l}
a_{n}=e^{i \theta_{1}}\left(z_{1} p_{n}+w_{1} f_{n}\right) \\
b_{n}=e^{i \theta_{1}}\left(-w_{1}^{*} p_{n}+z_{1}^{*} f_{n}\right)
\end{array}\right.
$$

Via Eq. (B1), the nonlinear terms become

$$
\begin{aligned}
& a_{n}\left|a_{n}\right|^{2}=e^{i \theta_{1}}\left[z_{1}\left|z_{1}\right|^{2} p_{n}\left|p_{n}\right|^{2}+z_{1}^{2} w_{1}^{*} p_{n}^{2} f_{n}^{*}+z_{1}^{*} w_{1}^{2} p_{n}^{*} f_{n}^{2}+w_{1}\left|w_{1}\right|^{2} f_{n}\left|f_{n}\right|^{2}+2\left|z_{1}\right|^{2} w_{1}\left|p_{n}\right|^{2} f_{n}+2 z_{1}\left|w_{1}\right|^{2} p_{n}\left|f_{n}\right|^{2}\right], \\
& b_{n}\left|b_{n}\right|^{2}=e^{i \theta_{1}}\left[-w_{1}^{*}\left|w_{1}\right|^{2} p_{n}\left|p_{n}\right|^{2}+z_{1} w_{1}^{* 2} p_{n}^{2} f_{n}^{*}-z_{1}^{* 2} w_{1} p_{n}^{*} f_{n}^{2}+z_{1}^{*}\left|z_{1}\right|^{2} f_{n}\left|f_{n}\right|^{2}+2 z_{1}^{*}\left|w_{1}\right|^{2}\left|p_{n}\right|^{2} f_{n}-2\left|z_{1}\right|^{2} w_{1}^{*} p_{n}\left|f_{n}\right|^{2}\right] .
\end{aligned}
$$

The equations for $p_{n}$ then read

$$
\begin{aligned}
i \dot{p}_{n}= & -\left(\left|z_{2}\right|^{2}-\left|w_{2}\right|^{2}\right) p_{n}+2 w_{2} z_{2} f_{n-1}+U\left\{\left(\left|z_{1}\right|^{4}+\left|w_{1}\right|^{4}\right) p_{n}\left|p_{n}\right|^{2}+2 z_{1}^{* 2} w_{1}^{2} p_{n}^{*} f_{n}^{2}+4\left|z_{1}\right|^{2}\left|w_{1}\right|^{2} p_{n}\left|f_{n}\right|^{2}\right. \\
& \left.+z_{1} w_{1}^{*}\left(\left|z_{1}\right|^{2}-\left|w_{1}\right|^{2}\right) p_{n}^{2} f_{n}^{*}+z_{1}^{*} w_{1}\left(\left|w_{1}\right|^{2}-\left|z_{1}\right|^{2}\right) f_{n}\left|f_{n}\right|^{2}+2 z_{1}^{*} w_{1}\left(\left|z_{1}\right|^{2}-\left|w_{1}\right|^{2}\right)\left|p_{n}\right|^{2} f_{n}\right\} .
\end{aligned}
$$

The unit-cell redefinition $f_{n} \longmapsto f_{n-1}$ yields the terms $f_{n-1}\left|f_{n-1}\right|^{2}$,

$$
\begin{aligned}
i \dot{p}_{n}= & -\left(\left|z_{2}\right|^{2}-\left|w_{2}\right|^{2}\right) p_{n}+2 w_{2} z_{2} f_{n}+U\left\{\left(\left|z_{1}\right|^{4}+\left|w_{1}\right|^{4}\right) p_{n}\left|p_{n}\right|^{2}+2 z_{1}^{* 2} w_{1}^{2} p_{n}^{*} f_{n-1}^{2}+4\left|z_{1}\right|^{2}\left|w_{1}\right|^{2} p_{n}\left|f_{n-1}\right|^{2}\right. \\
& \left.+z_{1} w_{1}^{*}\left(\left|z_{1}\right|^{2}-\left|w_{1}\right|^{2}\right) p_{n}^{2} f_{n-1}^{*}+z_{1}^{*} w_{1}\left(\left|w_{1}\right|^{2}-\left|z_{1}\right|^{2}\right) f_{n-1}\left|f_{n-1}\right|^{2}+2 z_{1}^{*} w_{1}\left(\left|z_{1}\right|^{2}-\left|w_{1}\right|^{2}\right)\left|p_{n}\right|^{2} f_{n-1}\right\},
\end{aligned}
$$

which break the caging effect. These terms are not present if $\left|w_{1}\right|^{2}=\left|z_{1}\right|^{2}$, which reduces Eq. (B4) to

$$
i \dot{p}_{n}=-\left(\left|z_{2}\right|^{2}-\left|w_{2}\right|^{2}\right) p_{n}+2 w_{2} z_{2} f_{n}+2 e^{i \theta_{1}} U\left\{\left|z_{1}\right|^{4}\left(p_{n}\left|p_{n}\right|^{2}+2 p_{n}\left|f_{n-1}\right|^{2}\right)+z_{1}^{* 2} w_{1}^{2} p_{n}^{*} f_{n-1}^{2}\right\} .
$$

The same holds true for $f_{n}$, where under the condition $\left|w_{1}\right|^{2}=\left|z_{1}\right|^{2}$ the motion equation reads

$$
i \dot{f}_{n}=\left(\left|z_{2}\right|^{2}-\left|w_{2}\right|^{2}\right) f_{n}+2 w_{2}^{*} z_{2}^{*} p_{n}+2 U\left\{\left|z_{1}\right|^{4}\left(f_{n}\left|f_{n}\right|^{2}+2\left|p_{n+1}\right|^{2} f_{n}\right)+z_{1}^{2} w_{1}^{* 2} p_{n+1}^{2} f_{n}^{*}\right\} .
$$

As a result, we observe the following:

(i) The condition $\left|w_{1}\right|^{2}=\left|z_{1}\right|^{2}$ in Eq. (A28) is necessary to preserve the caging-otherwise "fully nonlocal" terms that break the caging exist in Eq. (B4) — and it is sufficient—-since the subsequent transformation by $U_{2}$ in Eq. (A28) will not introduce additional "fully nonlocal" terms.

(ii) The condition $\left|w_{1}\right|^{2}=\left|z_{1}\right|^{2}$ in Eq. (A28) means that all the entries of the matrix $H_{1}$ in Eq. (A35) have equal magnitude in absolute value. 


\section{Rotating the interaction Hamiltonian $\mathcal{H}_{1}^{G}$}

The rotated Eqs. (B5) and (B6) are the equations of motion $i \dot{p}_{n}=\frac{\partial \mathcal{H}_{G}}{\partial p_{n}^{*}}$ and $i \dot{f}_{n}=\frac{\partial \mathcal{H}}{\partial f_{n}^{*}}$ of the Hamiltonian $\mathcal{H}_{G}$,

$$
\mathcal{H}_{G}=\mathcal{H}_{0}^{G}+\mathcal{H}_{1}^{G},
$$

where

$$
\mathcal{H}_{0}^{G}=\sum_{n}\left\{\left(\left|z_{2}\right|^{2}-\left|w_{2}\right|^{2}\right)\left(\left|f_{n}\right|^{2}-\left|p_{n}\right|^{2}\right)+2 w_{2} z_{2} p_{n}^{*} f_{n}+\text { H.c. }\right\}
$$

and

$$
\mathcal{H}_{1}^{G}=U \sum_{n}\left\{\left|z_{1}\right|^{4}\left[\left|p_{n}\right|^{4}+\left|f_{n}\right|^{4}+4\left|p_{n}\right|^{2}\left|f_{n+1}\right|^{2}\right]+z_{1}^{* 2} w_{1}^{2} p_{n}^{* 2} f_{n+1}^{2}+z_{1} w_{1}^{* 2} p_{n}^{2} f_{n+1}^{* 2}\right\} .
$$

\section{Fully detangled models $A$ and $B$}

By applying further transformations (see Fig. 1), one can fully detangle both models A and B introduced in the main text. This computation is straightforward but lengthy and we only provide the final result for both models. Namely, the nonlinear evolution equation for model $\mathrm{A}$ is

$$
\begin{aligned}
i \dot{\alpha}_{n}= & 2 \alpha_{n}+\frac{U}{8}\left\{\alpha_{n}\left|\alpha_{n}\right|^{2}+\beta_{n}^{2} \alpha_{n}^{*}+2 \alpha_{n}\left|\beta_{n}\right|^{2}+\alpha_{n}^{*} \alpha_{n+1}^{2}+\alpha_{n}^{*} \beta_{n+1}^{2}-2 \alpha_{n}^{*} \alpha_{n+1} \beta_{n+1}+\beta_{n}^{*} \alpha_{n+1}^{2}+\beta_{n}^{*} \beta_{n+1}^{2}-2 \beta_{n}^{*} \alpha_{n+1} \beta_{n+1}\right. \\
& +\alpha_{n}^{*} \alpha_{n-1}^{2}+\alpha_{n}^{*} \beta_{n-1}^{2}+2 \alpha_{n}^{*} \alpha_{n-1} \beta_{n-1}-\beta_{n}^{*} \alpha_{n-1}^{2}-\beta_{n}^{*} \beta_{n-1}^{2}-2 \beta_{n}^{*} \alpha_{n-1} \beta_{n-1}+2\left(\alpha_{n}\left|\alpha_{n+1}\right|^{2}-\alpha_{n} \alpha_{n+1}^{*} \beta_{n+1}\right. \\
& \left.-\alpha_{n} \alpha_{n+1} \beta_{n+1}^{*}+\alpha_{n}\left|\beta_{n+1}\right|^{2}\right)+2\left(\beta_{n}\left|\alpha_{n+1}\right|^{2}-\alpha_{n+1}^{*} \beta_{n} \beta_{n+1}-\alpha_{n+1} \beta_{n} \beta_{n+1}^{*}+\beta_{n}\left|\beta_{n+1}\right|^{2}\right) \\
& \left.+2\left(\alpha_{n}\left|\alpha_{n-1}\right|^{2}+\alpha_{n} \alpha_{n-1}^{*} \beta_{n-1}+\alpha_{n} \alpha_{n-1} \beta_{n-1}^{*}+\alpha_{n}\left|\beta_{n-1}\right|^{2}\right)-2\left(\beta_{n}\left|\alpha_{n-1}\right|^{2}+\alpha_{n-1}^{*} \beta_{n} \beta_{n-1}+\alpha_{n-1} \beta_{n} \beta_{n-1}^{*}+\beta_{n}\left|\beta_{n+1}\right|^{2}\right)\right\}
\end{aligned}
$$

and a similar equation for the other component $\beta_{n}$. It is straightforward to check that all the terms in the above expression are exactly of the type required by the nonlinear caging criterion (16) introduced in Sec. III A.

The equation for model B reads (again we only provide the equation for one component; the equation for the other component $\beta_{n}$ is qualitatively similar)

$$
\begin{aligned}
i \dot{\alpha}_{n}= & 4 \alpha_{n}+\frac{U}{64}\left\{2 \sqrt { 3 } \left[\alpha_{n+1}\left|\alpha_{n+1}\right|^{2}-\alpha_{n-1}\left|\alpha_{n-1}\right|^{2}-\alpha_{n+1}^{2} \beta_{n+1}^{*}-\alpha_{n-1}^{2} \beta_{n-1}^{*}+\beta_{n+1}^{2} \alpha_{n+1}^{*}-\beta_{n-1}^{2} \alpha_{n-1}^{*}\right.\right. \\
& \left.-\beta_{n+1}\left|\beta_{n+1}\right|^{2}-\beta_{n-1}\left|\beta_{n-1}\right|^{2}-2\left|\alpha_{n+1}\right|^{2} \beta_{n+1}-2\left|\alpha_{n-1}\right|^{2} \beta_{n-1}+2 \alpha_{n+1}\left|\beta_{n+1}\right|^{2}-2 \alpha_{n-1}\left|\beta_{n-1}\right|^{2}+[\cdots]\right\} .
\end{aligned}
$$

We have only kept the terms in the above expression that break the nonlinear caging criterion (16). The remaining terms, indicated by $[\cdots]$, conform with the caging criterion.

[1] P. W. Anderson, Absence of diffusion in certain random lattices, Phys. Rev. 109, 1492 (1958).

[2] B. Kramer and A. MacKinnon, Localization: theory and experiment, Rep. Prog. Phys. 56, 1469 (1993).

[3] D. M. Basko, I. L. Aleiner, and B. L. Altshuler, Metal-insulator transition in a weakly interacting many-electron system with localized single-particle states, Ann. Phys. 321, 1126 (2006).

[4] I. L. Aleiner, B. L. Altshuler, and G. V. Shlyapnikov, A finitetemperature phase transition for disordered weakly interacting bosons in one dimension, Nat. Phys. 6, 900 (2010).

[5] D. A. Abanin, E. Altman, I. Bloch, and M. Serbyn, Colloquium: Many-body localization, thermalization, and entanglement, Rev. Mod. Phys. 91, 021001 (2019).

[6] T. V. Laptyeva, M. V. Ivanchenko, and S. Flach, Nonlinear lattice waves in heterogeneous media, J. Phys. A 47, 493001 (2014)

[7] I. Vakulchyk, M. V. Fistul, and S. Flach, Wave Packet Spreading with Disordered Nonlinear Discrete-Time Quantum Walks, Phys. Rev. Lett. 122, 040501 (2019).
[8] A. Mielke, Ferromagnetic ground states for the hubbard model on line graphs, J. Phys. A 24, L73 (1991).

[9] H. Tasaki, Ferromagnetism in the Hubbard Models with Degenerate Single-Electron Ground States, Phys. Rev. Lett. 69, 1608 (1992).

[10] O. Derzhko, J. Richter, and M. Maksymenko, Strongly correlated flat-band systems: The route from heisenberg spins to hubbard electrons, Int. J. Mod. Phys. B 29, 1530007 (2015)

[11] D. Leykam, A. Andreanov, and S. Flach, Artificial flat band systems: from lattice models to experiments, Adv. Phys.: X 3, 1473052 (2018).

[12] D. Leykam and S. Flach, Perspective: Photonic flatbands, APL Photon. 3, 070901 (2018).

[13] J. Vidal, R. Mosseri, and B. Douçot, Aharonov-Bohm Cages in Two-Dimensional Structures, Phys. Rev. Lett. 81, 5888 (1998).

[14] B. Douçot and J. Vidal, Pairing of Cooper Pairs in a Fully Frustrated Josephson-Junction Chain, Phys. Rev. Lett. 88, 227005 (2002). 
[15] K. Fang, Z. Yu, and S. Fan, Photonic Aharonov-Bohm Effect Based on Dynamic Modulation, Phys. Rev. Lett. 108, 153901 (2012).

[16] S. Longhi, Aharonov-Bohm photonic cages in waveguide and coupled resonator lattices by synthetic magnetic fields, Opt. Lett. 39, 5892 (2014).

[17] O. V. Kibis, H. Sigurdsson, and I. A. Shelykh, Aharonov-Bohm effect for excitons in a semiconductor quantum ring dressed by circularly polarized light, Phys. Rev. B 91, 235308 (2015).

[18] M. Hasan, I. V. Iorsh, O. V. Kibis, and I. A. Shelykh, Optically controlled periodical chain of quantum rings, Phys. Rev. B 93, 125401 (2016).

[19] K. Fang, Z. Yu, and S. Fan, Realizing effective magnetic field for photons by controlling the phase of dynamic modulation, Nat. Photon. 6, 782 (2012).

[20] S. Mukherjee, M. Di Liberto, P. Öhberg, R. R. Thomson, and N. Goldman, Experimental Observation of Aharonov-Bohm Cages in Photonic Lattices, Phys. Rev. Lett. 121, 075502 (2018).

[21] S. Gladchenko, D. Olaya, E. Dupont-Ferrier, B. Douçot, L. B. Ioffe, and M. E. Gershenson, Superconducting nanocircuits for topologically protected qubits, Nat. Phys. 5, 48 (2009).

[22] G. Gligorić, P. P. Beličev, D. Leykam, and A. Maluckov, Nonlinear symmetry breaking of Aharonov-Bohm cages, Phys. Rev. A 99, 013826 (2019).

[23] M. Di Liberto, S. Mukherjee, and N. Goldman, Nonlinear dynamics of Aharonov-Bohm cages, Phys. Rev. A 100, 043829 (2019).

[24] J. Vidal, B. Douçot, R. Mosseri, and P. Butaud, Interaction Induced Delocalization for Two Particles in a Periodic Potential, Phys. Rev. Lett. 85, 3906 (2000).

[25] M. Creutz, End States, Ladder Compounds, and Domain-Wall Fermions, Phys. Rev. Lett. 83, 2636 (1999).

[26] S. Takayoshi, H. Katsura, N. Watanabe, and H. Aoki, Phase diagram and pair Tomonaga-Luttinger liquid in a Bose-Hubbard model with flat bands, Phys. Rev. A 88, 063613 (2013).

[27] M. Tovmasyan, E. P. L. van Nieuwenburg, and S. D. Huber, Geometry-induced pair condensation, Phys. Rev. B 88, 220510(R) (2013).

[28] M. Tovmasyan, S. Peotta, P. Törmä, and S. D. Huber, Effective theory and emergent SU(2) symmetry in the flat bands of attractive Hubbard models, Phys. Rev. B 94, 245149 (2016).

[29] J. Jünemann, A. Piga, S.-J. Ran, M. Lewenstein, M. Rizzi, and A. Bermudez, Exploring Interacting Topological Insulators with Ultracold Atoms: The Synthetic Creutz-Hubbard Model, Phys. Rev. X 7, 031057 (2017).

[30] M. Tovmasyan, S. Peotta, L. Liang, P. Törmä, and S. D. Huber, Preformed pairs in flat bloch bands, Phys. Rev. B 98, 134513 (2018).

[31] J. Vidal, P. Butaud, B. Douçot, and R. Mosseri, Disorder and interactions in Aharonov-Bohm cages, Phys. Rev. B 64, 155306 (2001).

[32] C. Danieli, A. Andreanov, T. Mithun, and S. Flach, Quantum caging in interacting many-body all-bands-flat lattices, Phys. Rev. B 104, 085132 (2021).

[33] N. Read, Compactly supported Wannier functions and algebraic $k$-theory, Phys. Rev. B 95, 115309 (2017).
[34] S. Flach, D. Leykam, J. D. Bodyfelt, P. Matthies, and A. S. Desyatnikov, Detangling flat bands into Fano lattices, Europhys. Lett. 105, 30001 (2014).

[35] F. Baboux, L. Ge, T. Jacqmin, M. Biondi, E. Galopin, A. Lemaître, L. Le Gratiet, I. Sagnes, S. Schmidt, H. E. Türeci, A. Amo, and J. Bloch, Bosonic Condensation and DisorderInduced Localization in a Flat Band, Phys. Rev. Lett. 116, 066402 (2016).

[36] B. Real, C. Cantillano, D. López-González, A. Szameit, M. Aono, M. Naruse, S.-J. Kim, K. Wang, and R. A. Vicencio, Flat-band light dynamics in stub photonic lattices, Sci. Rep. 7, 15085 (2017).

[37] D. L. Bergman, C. Wu, and L. Balents, Band touching from real-space topology in frustrated hopping models, Phys. Rev. B 78, 125104 (2008).

[38] R. G. Dias and J. D. Gouveia, Origami rules for the construction of localized eigenstates of the Hubbard model in decorated lattices, Sci. Rep. 5, 16852 (2015).

[39] L. Morales-Inostroza and R. A. Vicencio, Simple method to construct flat-band lattices, Phys. Rev. A 94, 043831 (2016).

[40] W. Maimaiti, A. Andreanov, H. C. Park, O. Gendelman, and S. Flach, Compact localized states and flat-band generators in one dimension, Phys. Rev. B 95, 115135 (2017).

[41] M. Röntgen, C. V. Morfonios, and P. Schmelcher, Compact localized states and flat bands from local symmetry partitioning, Phys. Rev. B 97, 035161 (2018).

[42] L. A. Toikka and A. Andreanov, Necessary and sufficient conditions for flat bands in m-dimensional $n$-band lattices with complex-valued nearest-neighbour hopping, J. Phys. A 52, 02LT04 (2018).

[43] W. Maimaiti, S. Flach, and A. Andreanov, Universal $d=1$ flat band generator from compact localized states, Phys. Rev. B 99, 125129 (2019).

[44] F. D. M. Haldane, Model for a Quantum Hall Effect Without Landau Levels: Condensed-Matter Realization of the "Parity Anomaly," Phys. Rev. Lett. 61, 2015 (1988).

[45] Consider a gauge transformation $\mathcal{G}:\left\{a_{n}, b_{n}\right\} \mapsto\left\{e^{i \xi_{n}} a_{n}, e^{i \eta_{n}} b_{n}\right\}$ defined for arbitrary phase functions $\xi_{n}, \eta_{n}$. The submanifold of lattices related to the Creutz ladder via $\mathcal{G}$ is given by the family of parameters $z_{1}=e^{i \frac{\xi_{n}-\eta_{n}}{2}} / \sqrt{2}, w_{1}=i e^{i \frac{i \eta_{n}-\eta_{n}}{2}} / \sqrt{2}, z_{2}=$ $e^{i g_{n}\left(\xi_{n}, \eta_{n}\right)} / \sqrt{2}, w_{2}=e^{-i g_{n}\left(\xi_{n}, \eta_{n}\right)} / \sqrt{2}$ in Eqs. (5) and (6) for arbitrary functions $g_{n}$.

[46] One could potentially imagine further fine-tuning of the interaction that might cancel the total sum but not the individual terms: this is, however, only possible for a specific combination of the amplitudes $\phi_{n, a}$, implying that at best only specific excitations can be caged. We are interested in caging of any initial excitation and therefore we do not investigate this possibility any further.

[47] C. Danieli, B. M. Manda, T. Mithun, and Ch. Skokos, Computational efficiency of numerical integration methods for the tangent dynamics of many-body hamiltonian systems in one and two spatial dimensions, Math. Eng. 1, 447 (2019).

[48] M. Röntgen, C. V. Morfonios, I. Brouzos, F. K. Diakonos, and P. Schmelcher, Quantum Network Transfer and Storage with Compact Localized States Induced by Local Symmetries, Phys. Rev. Lett. 123, 080504 (2019). 